\title{
EXPONENTIAL DIOPHANTINE EQUATIONS
}

\author{
J. L. Brenner and Lorraine L. Foster
}

\section{Contents}

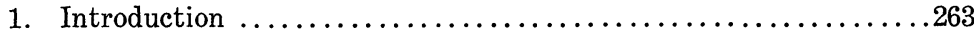

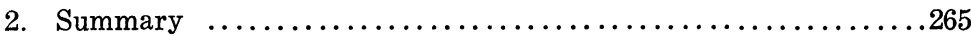

3. Equations of the Form $1+x^{a}=y^{b}+z^{c} \ldots \ldots \ldots \ldots \ldots \ldots \ldots 266$

4. Equations of the form $x^{a}+y^{b}=z^{c}+w^{d} \ldots \ldots \ldots \ldots \ldots \ldots . \ldots 269$

5. Theorems Concerning Equations of the General Form

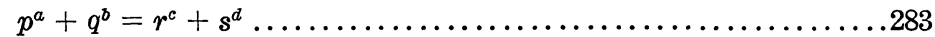

6. The Equations $1+2^{a}+7^{b}=3^{c}+5^{d}, 3^{a}+7^{b}=3^{c}+5^{d}+2 \ldots \ldots 286$

7. Equations of the Form $p^{a}+q^{b}+r^{c}=s^{d} \ldots \ldots \ldots \ldots \ldots \ldots \ldots 294$

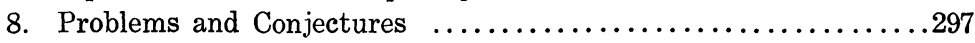

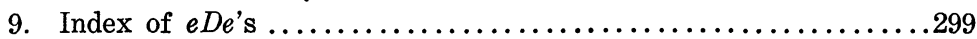

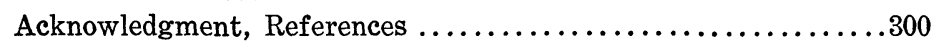

We study equations in which the unknowns are the exponents. (Work in this field originated with C. St $\not$ rmer and D. H. Lehmer. More recently, Leo J. Alex has extended their results; his work relates to classification of nonabelian simple groups.)

(i) For the equation $k+7^{a}=3^{c}+5^{d}, k=3^{b}, 5^{b}, 13^{b}$, or $17^{b}$, and for many similar 4 -term equations, we find all integral solutions.

(ii) We find all integral solutions of $3^{a}+7^{b}=3^{c}+5^{d}+2$.

(iii) We prove that there are infinitely many odd $m$ such that $m^{a}+7^{b}=3^{c}+5^{d}$ has only the solutions $(a, b, c, d)=$ $(0,0,0,0),(0,1,1,1)$.

1. Introduction. By an exponential Diophantine equation $(e D e)$ we mean an equation in which the bases are (given or unknown) integers; the exponents are unknown integers. In this article the exponents are nonnegative as well. Examples are the equations

$$
\begin{aligned}
& 1+2^{a}+7^{b}=3^{c}+5^{d}, \\
& 3^{a}+7^{b}=3^{c}+5^{d}+2 .
\end{aligned}
$$

(All solutions of these equations are determined in $\S 6$ of this article.)

Isolated examples of $e D e$ 's occur very early in the history of the theory of numbers (Mersenne, Fermat). The equation $x^{y}=y^{x}$ is another hoary example. To compute accurately the logarithms of primes, Størmer [12] found all solutions of the equation $1+2^{a} 3^{b} 5^{c}=$ $2^{d} 3^{e} 5^{f}$; his method depended on the theory of Pell equations. Lehmer [8] refined Størmer's methods. This approach is still useful for computing the logarithm of a prime to high precision. 
Another application [2a] of $e D e^{\prime} \mathrm{s}$ is to the theory of finite nonabelian simple groups. Suppose $G$ is such a group, $p, q_{1}, \cdots, q_{s}$ are the distinct primes dividing $|G|, \quad p^{2} \nmid|G|$, and $m=[N: C]$ where $N, C$ are respectively the normalizer and centralizer in $G$ of a Sylow $p$-subgroup, $S_{p}$. Then the degree equation for a $p$-block of highest defect must have the form

$$
\sum_{i=1}^{m} \pm x_{i}^{a(i)}=0,
$$

where $q_{1}, \cdots, q_{s}$ are the only possible prime factors of $x_{i}, i=$ $1,2, \cdots, m$. (If one of the terms of (1.01) is 1 , the $p$-block involved is the principal $p$-block.) One step in the solution of (1.01) is to find all solutions when the $x_{i}$ are themselves primes. In particular the finite nonabelian simple groups of order $2^{a} 3^{b} 7^{c} p$ and $2^{a} 3^{b} 5^{c} 7^{d} p$ with $m=2$ and $C=S_{p}$ were classified by L. J. Alex [2c, d] using $e D e$ 's.

If the conditions in equation (1.01) are changed to read: the prime factors of $x_{i}$ are all contained in the finite set $M_{i}$, and $M_{i} \cap$ $M_{j}=\varnothing$ if $i \neq j$, then it is known [11] that there are only finitely many solutions. For certain problems of this type we find all solutions by elementary methods; but we cannot prove that elementary methods always suffice to this end, even when $M_{i}=\left\{q_{i}\right\}, q_{i}$ a single prime. Here "elementary methods" means reduction of (1.01) to a congruence with respect to single (perhaps a large) modulus.

We note especially that we find all solutions in several cases to which the general results of [11] do not apply at all. But characterization of those equations solvable by elementary methods has eluded us.

A third application is to the study of the class equation for a finite group $G$. If $g$ is the order of $G$, then

$$
g=1+h_{1}+\cdots+h_{c},
$$

where $c$ is the number of nontrivial classes, and where $h_{i}$ is the cardinality of the $i$ th class. It is known that $h_{i}$ divides $g$, so that for each $c$, and for a given $g$, (1.02) is an $e D e$. For fixed $g$, the equation clearly has only a finite number of solutions. It is interesting to find the solutions (for given $g$ ) for each $c$. If $x_{i}\left(x_{i} \mid g\right)$ satisfy

$$
g=1+x_{1}+\cdots+x_{c},
$$

it is usually a separate question to decide whether a finite group with $c$ nontrivial classes $C_{i}$ exists with $\left|C_{i}\right|=x_{i}$. (If $g$ is too small, there is no solution to (1.02); this puts a bound on the order of a group with a preassigned number of classes.) 
An index of $e D e$ 's is given in $\S 9$ of this article. When confronted with a new $e D e$, one should first use parity checks; if all solutions are not immediately apparent, one may nevertheless find that a parity check will reduce the problem to one of the problems in our index. Again, by choosing special values of certain exponents in the equations in the index, one may be able to subsume the equation in question under an indexed equation. Finally, if neither of these approaches is effective, it may be necessary to apply our methods (if not our results) in some detail. Hence, a fairly complete explanation of the methods is given in the body of the paper.

We have some comments about these methods. With a few classes of exceptions, we have been able to find all solutions of each equation studied in this article by applying elementary methods exclusively. This is not to say that the approach is easy: in some cases our goal was reached only after expending energy comparable to that needed to hike long distances through a heavy snowstorm. Our work has led us to certain conjectures (see §8).

2. Summary. This article is separated into several sections, as indicated in the table of contents (which appears immediately following the abstract). The interconnection of the various sections is as follows. Section 3 is concerned primarily with rather easy examples of exponential Diophantine equations of the form $1+x^{a}=$ $y^{b}+z^{c}$, where $x, y, z$ are given. All of these equations are completely solved by the use of modular arithmetic. Some generalizations of these equations are considered in $\S 4$. Here we adhere to the same methods but the difficulties encountered are much greater. In fact, in some cases, for brevity, we refer to the rather potent results of $\S 6$ (which is independent of the other sections). The logical sequence of sections is thus different from the actual progression that appears. For, we have anticipated that some readers might prefer to peruse the material without concerning themselves with the truth of all statements. We emphasize that when we claim to find all solutions of an eDe, all of the arguments to support our claim are given in full in this article. (However, exceedingly trivial proofs are omitted entirely.)

In $\S 8$ we note that some $e D e^{\prime}$ 's cannot be solved using modular arithmetic and a finite number of moduli. The equation $3^{a}+7^{b}=$ $3^{c}+5^{d}$ can be solved completely (i.e., all integer solutions can be found, with proof that there are no others) using a finite number of moduli. On the other hand, it is not clear how to find all solutions of the equation $3^{a}+5^{b}=3^{c}+5^{d}$; we can however prove that no finite number of moduli is sufficient for this purpose.

In several sections, in particular in $\S 5$, we give examples of 
infinite sets of $e D e$ 's that can be solved using only a finite number of moduli. For certain infinite sets of $e D e$ 's we show that no nontrivial solutions exist. (A solution is called trivial if all exponents are zero.) On the other hand, there are infinitely many $e D e$ 's of the form $1+(p q)^{a}=p^{b}+q^{c}$ which cannot be solved using a finite number of moduli.

The problems in $\S 7$ vary considerably in difficulty. Further, it is easy to invent equations of the form of this section that have no solution.

Additional problems (without solutions) and conjectures are presented in $\S 8$. In $\S 9$ we attempt to list all $e D e$ 's with which we are familiar (in addition to those solved in this article).

3. Equations of the Form $1+x^{a}=y^{b}+z^{c}$. Some equations of the form considered in this section (see [5]) provided the motivation for this paper. Those included here are rather easy to solve. Further, each of these equations (with $x, y, z$ fixed) can undoubtedly be generalized in many ways to an equation of the form $X^{a}+$ $Y^{b}=Z^{c}+W^{d}$.

\subsection{THEOREM. The solutions of}

$$
1+2^{a}=2^{b}+3^{c}
$$

in nonnegative integers are: $(a, b, c)=(2,1,1),(4,3,2)$ or $(t, t, 0), t$ arbitrary.

Proof. Let $(a, b, c)$ be another solution. Clearly $c>0$. Using $\bmod 3$ we find that $a$ is even, $b$ is odd. Thus, using $\bmod 8, b \geqq 3$ and $c$ is even. Either $b=3$ or $b \geqq 4$. Suppose $b \geqq 4$. Then using $\bmod 16, c \equiv 0(\bmod 4)$ so that $2^{a} \equiv 2^{b}(\bmod 5)$, a contradiction. Hence, $b=3,2^{a}=7+3^{c}$. Clearly $c \geqq 4$. Therefore, using $\bmod 81, a \equiv 16$ $(\bmod 54)$. Using $\bmod 19, c \equiv 16(\bmod 18)$. Thus we have a contradiction mod 109 and there is no other solution.

\subsection{THEOREM. The solutions to}

$$
1+2^{a}=2^{b}+5^{c}
$$

\begin{tabular}{|ccc|}
\hline$a$ & $b$ & $c$ \\
\hline$t$ & $t$ & 0 \\
3 & 2 & 1 \\
5 & 3 & 2 \\
7 & 2 & 3 \\
\hline
\end{tabular}

3.05 Table. The solutions of (3.04). 
in nonnegative integers are given in Table 3.05. Here $t$ is arbitrary.

Proof. Let $(a, b, c)$ be another solution. Clearly $a, b, c>0$. Since $5^{c}-1=2^{b}\left(2^{a-b}-1\right)$, examining cases we find that $c \geqq 4$ so that $a \geqq 10$. Using mod 16 we conclude that $b>1$. We consider three cases: $b=2, b=3$ and $b \geqq 4$.

3.051 Case. $b=2$. Thus $2^{a}=3+5^{c}, 5^{c} \equiv-3(\bmod 1024), c \equiv$ $163(\bmod 256)$. Thus we have a contradiction $\bmod 257$.

3.052 Case. $b=3$. Thus $2^{a}=7+5^{c}, 5^{c} \equiv-7(\bmod 64), c \equiv 10$ ( $\bmod 16)$. Then, using $\bmod 3, a$ is odd. This yields a contradiction $\bmod 17$.

3.053 Case. $b \geqq 4$. Then $5^{c} \equiv 1(\bmod 16)$ so that $c \equiv 0(\bmod 4)$. Hence, using $\bmod 13, a \equiv b(\bmod 12)$. Thus we have a contradiction $\bmod 5$.

\subsection{THEOREM. The only solutions to}

$$
1+2^{a}=2^{b}+7^{c}
$$

in nonnegative integers are $(a, b, c)=(3,3,1),(6,4,2)$ or $(t, t, 0), t$ arbitrary.

Proof. Let $(a, b, c)$ be another solution. Clearly $b, c>0$. Since $7^{c}-1=2^{b}\left(2^{a-b}-1\right)$ we can easily verify that $c \geqq 4, a>11$. Further using $\bmod 7,(a, b) \equiv(0,1)(\bmod 3)$. If $b=1$ we immediately have a contradiction mod 16. Also, if $b=2$ or 3 we have a contradiction $\bmod 7$. Hence we have two remaining cases: $b=4$ and $b \geqq 5$.

3.071 Case. $b=4$. Then $2^{a}=15+7^{c}$. Hence $7^{\circ} \equiv 113(\bmod 128)$ so that $c \equiv 10(\bmod 16)$. Thus $2^{a} \equiv 15+2 \equiv 0(\bmod 17)$, a contradiction.

3.072 Case. $b \geqq 5$. Thus $1 \equiv 7^{\circ}(\bmod 32)$ so that $c \equiv 0(\bmod 4)$. Then, using $\bmod 9,(a, b, c) \equiv(3,1,1)$ or $(0,4,2)(\bmod (6,6,3))$, so that, in fact $(a, b, c) \equiv(3,1,4)$ or $(0,4,8)(\bmod (6,6,12))$. Using $\bmod 13$ we conclude that $(a, b, c) \equiv(6,10,8)(\bmod 12)$. Examining cases $\bmod 19$ we conclude that $(a, b) \equiv(6,22)(\bmod 36)$. This yields a contradiction $\bmod 37$.

3.08 THEOREM. The only solutions of

$$
1+5^{a}=3^{b}+3^{c}
$$


in nonnegative integers are $(a, b, c)=(0,0,0)$ or $(1,1,1)$.

Proof. Let $(a, b, c)$ be another solution. Clearly $a b c \neq 0$. Hence using $\bmod 3, a$ is odd, so that, using $\bmod 8, b$ and $c$ are also odd. Either $b=1$ or $b>1$. Suppose $b=1$. Then $c>1$ so that, using $\bmod 9, a \equiv 5(\bmod 6)$. Thus, using $\bmod 7, c \equiv 0(\bmod 6)$ and we have a contradiction mod 13. Hence $b>1$ and by symmetry $c>1$. Thus from $\bmod 9, a \equiv 3(\bmod 6)$. This yields a contradiction $\bmod 7$.

\subsection{THEOREM. The only solutions to}

$$
1+5^{a}=3^{b}+23^{c}
$$

in nonnegative integers are $(a, b, c)=(0,0,0)$ or $(2,1,1)$.

Proof. Let $(a, b, c)$ be another solution. Clearly $a b c \neq 0$. Using $\bmod 8$ we conclude that $a \equiv 0, b \equiv c(\bmod 2)$. Hence, using the moduli 5,16 successively we have $b \equiv c \equiv 1, a \equiv 2(\bmod 4)$. We consider two cases: $b=1$ and $b \geqq 2$.

3.111 Case. $b=1$. Then $5^{a}=2+23^{c}$. Clearly $a>2$ so that $23^{c} \equiv-2(\bmod 125)$. Hence $c \equiv 21(\bmod 100), 5^{a} \equiv 66(\bmod 101)$, a contradiction.

3.112 Case. $b \geqq 2$. Then $1+5^{a} \equiv 5^{c}(\bmod 9)$ so that $(a, c) \equiv$ $(0,5),(2,3)$ or $(4,1)(\bmod 6)$. Using $\bmod 13, b \equiv c(\bmod 6)$, so that, using $\bmod 7,(a, b, c) \equiv(0,5,5)(\bmod 6)$. Thus, using $\bmod 19,(a, b, c) \equiv$ $(3,5,2)$ or $(6,11,5)(\bmod (9,18,9))$ so that in fact $(a, b, c) \equiv(30,5,29)$ or $(6,29,5)(\bmod 36)$. Thus we have a contradiction $\bmod 37$.

\subsection{THEOREM. The only solutions of}

$$
1+5^{a}=7^{b}+19^{c}
$$

in nonnegative integers are $(a, b, c)=(0,0,0)$ and $(2,1,1)$.

Proof. Let $(a, b, c)$ be another solution. Clearly $b, c>0, a \geqq 3$. Using $\bmod 3, a$ is even. Further, using $\bmod 5, b$ and $c$ are odd. Thus, using $\bmod 7$ we conclude $(a, c) \equiv(2,1)$ or $(4,5)(\bmod 6)$, so that, using $\bmod 9,(a, b, c) \equiv(2,1,1)$ or $(4,5,5)(\bmod 6)$. We consider two cases: $b=1$ and $b \geqq 2$.

3.131 Case. $b=1$. Then $5^{a}=6+19^{c}$. Using $\bmod 125$ we find that $c \equiv 31(\bmod 50)$ so that using $\bmod 11, a \equiv 2(\bmod 5)$. Thus we have a contradiction $\bmod 101$. 
3.132 Case. $b \geqq 2$. Thus $1+5^{a} \equiv 19^{\circ}(\bmod 49)$ so that $(a, b, c) \equiv$ $(14,1,1)$ or $(28,5,5)(\bmod (42,6,6))$. In each case there is a contradiction $\bmod 43$.

The following theorem deals with several very easy examples of the form $1+p^{a}=q^{b}+q^{c}, p, q$ distinct primes.

3.14 THEOREM. The only solution to each of the following equations in nonnegative integers is $(a, b, c)=(0,0,0)$ :

$$
\begin{aligned}
& 1+5^{a}=7^{b}+7^{c} \\
& 1+5^{a}=11^{b}+11^{c} \\
& 1+5^{a}=17^{b}+17^{c} \\
& 1+13^{a}=17^{b}+17^{c} \\
& 1+17^{a}=7^{b}+7^{c} \\
& 1+67^{a}=17^{b}+17^{c} .
\end{aligned}
$$

Proof. (3.141) Use mod 7 and mod 3. (3.142) Use mod 11. (3.143) Use the moduli 8,3 and 5 successively. (3.144) Use $\bmod 17$ and $\bmod 16$. (3.145) Use $\bmod 7$ and $\bmod 9$. (3.146) Use $\bmod 17$ and $\bmod 8$.

We conclude this section with an obvious general theorem:

3.15 THEOREM. Let $p, q, r$ be distinct primes such that $q \equiv 1$ $(\bmod p)$. Then

$$
1+p^{a}=q^{b}+r^{c}
$$

has only the trivial solution.

4. Equations of the Form $x^{a}+y^{b}=z^{c}+w^{d}$. In this section we find all solutions in nonnegative integers of several equations with four variable exponents. While some of these are quite easy, others are rather difficult.

\subsection{THEOREM. The solutions to}

$$
3^{a}+7^{b}=3^{c}+5^{d}
$$

in nonnegative integers are given in Table (4.03). Here $t$ is arbitrary. 


\begin{tabular}{|cccc|}
\hline$a$ & $b$ & $c$ & $d$ \\
\hline$t$ & 0 & $t$ & 0 \\
1 & 1 & 2 & 0 \\
0 & 1 & 1 & 1 \\
1 & 2 & 3 & 2 \\
3 & 0 & 1 & 2 \\
3 & 1 & 2 & 2 \\
\hline
\end{tabular}

Proof. Suppose $(a, b, c, d)$ is another solution. Referring to Theorem 6.01 we conclude that $a \neq 1,2$. Further from [5], $a \neq 0$ so that in fact $a \geqq 3$. Using mod 3, clearly $c>0$. The theorem is a consequence of the following five lemmas:

\subsection{Lemma. $b>0$.}

Proof. Suppose $b=0$. Then $3^{a}+1=3^{c}+5^{d}$. Clearly $d>0$. We consider two cases: $c=1$ and $c>1$.

4.0111 Case. $c=1$. Then $3^{a}=2+5^{c}$. Referring to Theorem 6.05 (with $b=c=0$ ) we have a contradiction. Hence $c>1$.

4.0112 Case. $c>1$. Then $1 \equiv 5^{d}(\bmod 9)$ so that $d \equiv 0(\bmod 6)$. Thus, using $\bmod 7, a \equiv c(\bmod 6)$. Hence, using $\bmod 13, d \equiv 0(\bmod 4)$ so that $d \equiv 0(\bmod 12)$. Further, using $\bmod 5$ we conclude that $a \equiv 3, c \equiv 1(\bmod 4)$. Also, using $\bmod 31$ we conclude that $a \equiv c$ $(\bmod 30)$. Thus $1 \equiv 5^{d}(\bmod 11)$ so that $d \equiv 0(\bmod 5)$. In particular $d \equiv 0(\bmod 20)$ so that using $\bmod 41$ we conclude $a \equiv c(\bmod 8)$, a contradiction. Hence $b>0$.

4.012 Lemma. $d>0$.

Proof. Assume the contrary. Then $3^{a}+7^{b}=3^{c}+1$. Clearly $c>1$. Hence $7^{b} \equiv 1(\bmod 9), b \equiv 0(\bmod 3)$. Thus, using $\bmod 19$, $a \equiv c(\bmod 18)$. This yields a contradiction $\bmod 7$.

4.013 LemMa. $c>1$.

Proof. Assume $c=1$. Then $3^{a}+7^{b}=3+5^{d}=3^{0}+5^{d}+2$. Thus from Theorem 6.05 we have a contradiction.

4.014 Lemma. $(a, b, d) \equiv(3,1,2)(\bmod 36)$ and $c=2$.

Proof. Since $7^{b} \equiv\left(5^{2}\right)^{b} \equiv 5^{d}(\bmod 9)$, we conclude that $d$ is even. 
Using the moduli 5, 16 successively we have the following table:

\begin{tabular}{|cccc|}
\hline$a$ & $b$ & $c$ & $d$ \\
\hline 1 & 2 & 3 & 2 \\
1 & 3 & 0 & 2 \\
3 & 0 & 1 & 2 \\
3 & 1 & 2 & 2 \\
\hline
\end{tabular}

4.04 Table. $(a, b, c, d) \bmod 4$.

In particular, $d \equiv 2(\bmod 4), a$ is odd and $b \neq \equiv c(\bmod 2)$. Thus, considering the moduli 7 and 9 successively we have the following table:

\begin{tabular}{|llll|}
\hline$a$ & $b$ & $c$ & $d$ \\
\hline 1 & 5 & 0 & 4 \\
1 & 3 & 2 & 0 \\
1 & 4 & 3 & 2 \\
3 & 0 & 5 & 0 \\
3 & 1 & 2 & 2 \\
3 & 5 & 4 & 4 \\
5 & 3 & 4 & 0 \\
5 & 1 & 0 & 2 \\
5 & 2 & 1 & 4 \\
\hline
\end{tabular}

4.05 Table. $(a, b, c, d) \bmod 6$.

Combining the above results and using mod 13 we conclude that $(a, b, c, d) \equiv(1,3,2,0)$ or $(3,1,2,2)(\bmod (6,12,6,6))$. Thus $(a, b, c, d) \equiv$ $(1,3,8,6)$ or $(3,1,2,2)(\bmod 12)$. Using $\bmod 19$ we conclude $(a, b, c, d) \equiv$ $(13,0,14,3),(3,1,2,2)$ or $(3,1,8,5)(\bmod (18,3,18,9))$ so that in fact $(a, b, c, d) \equiv(13,3,32,30),(3,1,2,2)$ or $(3,1,26,14)(\bmod (36,12,36,36))$. Finally, using $\bmod 37$ we conclude $(a, b, c, d) \equiv(3,1,2,2)(\bmod 36)$. Now suppose $c \geqq 3$. Then, since $b \equiv 1(\bmod 9)$ we have $7 \equiv 5^{d}$ $(\bmod 27)$ so that $d \equiv 14(\bmod 18)$, a contradiction. Hence $(a, b, d) \equiv$ $(3,1,2)(\bmod 36)$ and $c=2$.

4.055 Lemma. $c \neq 2$. (Thus $(a, b, c, d)$ does not exist.)

Proof. Suppose on the contrary that $c=2$. Then $3^{a}+7^{b}=$ $9+5^{d}$. We consider two cases: $b=1, b>1$.

4.0551 Case. $b=1$. Then $3^{a}=2+5^{d}$ so that $3^{a}+7^{0}=$ $3^{0}+5^{d}+2$. Hence referring to Theorem 6.05 we have a contradiction. 
4.0552 Case. $b>1$. Then $3^{a} \equiv 9+5^{d}(\bmod 49)$ so that $(a, d) \equiv$ $(3,14),(9,2),(15,32),(21,20),(27,8),(33,38)$ or $(39,26)(\bmod 42)$. In each case we have a contradiction $\bmod 43$.

4.06 THEOREM. The solutions to

$$
5^{a}+7^{b}=3^{c}+5^{d}
$$

in nonnegative integers are given in the following table. Here $t$ is arbitrary.

\begin{tabular}{|cccc|}
\hline$a$ & $b$ & $c$ & $d$ \\
\hline$t$ & 0 & 0 & $t$ \\
0 & 1 & 1 & 1 \\
2 & 1 & 3 & 1 \\
2 & 3 & 5 & 3 \\
\hline
\end{tabular}

4.08 Table. The solutions of (4.07).

Proof. Let $(a, b, c, d)$ be another solution. From [5], $a, d>0$. Further, from 6.01, $a>1$. The remainder of the proof consists of the following seven lemmas:

\subsection{LEMMA. $b>0$.}

Proof. Suppose $b=0$. Then $5^{a}+1=3^{c}+5^{d}$. Clearly $c>0$. Hence, using $\bmod 3, a \equiv 0, d \equiv 1(\bmod 2)$. Thus we have a contradiction $\bmod 8$.

\subsection{LEMMA. $c>0$.}

Proof. Suppose $c=0$. Then $5^{a}+7^{b}=1+5^{d}$. Thus $7^{b} \equiv 1$ $(\bmod 5)$ so that $b \equiv 0(\bmod 4)$. Using $\bmod 3, a \equiv d(\bmod 2)$. Examining cases $\bmod 7$ we conclude $(a, d) \equiv(3,1)$ or $(4,0)(\bmod 6)$. In either case, using $\bmod 9$ we conclude that $b \equiv 1(\bmod 3)$ so that in fact $b \equiv 4(\bmod 12)$. Applying these results $\bmod 13$ we have a contradiction.

\subsection{LEMMA. $d>1$.}

Proof. Suppose $d=1$. Then $5^{a}+7^{b}=3^{c}+5$. Clearly $c \geqq 4$. Using $\bmod 3$ we conclude that $a$ is even. Hence, using $\bmod 8, b$ and $c$ are odd. Thus, using the moduli 7 and 9 successively we have $(a, b, d) \equiv(0,5,1)$ or $(2,1,3)(\bmod 6)$. Applying the above results $\bmod 13$ we conclude that $(a, b, c) \equiv(2,1,3)(\bmod (12,12,6)) . \quad$ If $b=1$ 
then $5^{a}+2=3^{c}$ and we have a contradiction by Theorem 6.05. Hence $b>1$. Thus $5^{a} \equiv 3^{c}+5(\bmod 49)$. Hence $(a, c) \equiv(2,39)$, $(8,15),(14,33),(20,9),(26,27),(32,3)$ or $(38,21)(\bmod 42)$. In each case we have a contradiction $\bmod 43$. Hence $d>1$.

\subsection{Lemma. $(a, b, c, d) \equiv(2,1,3,1)$ or $(2,3,5,3)(\bmod 36)$.}

Proof. If $c=1$ then $7^{b} \equiv 3(\bmod 25)$, an absurdity. Hence $c>1$. Using the moduli 3 and 8 successively we conclude that $a \equiv 0, b \equiv$ $c \equiv d \equiv 1(\bmod 2)$. Thus, using the moduli 7 and 9 successively we have the following table:

\begin{tabular}{|cccc|}
\hline$a$ & $b$ & $c$ & $d$ \\
\hline 0 & 5 & 1 & 1 \\
0 & 3 & 5 & 5 \\
2 & 1 & 3 & 1 \\
2 & 3 & 5 & 3 \\
4 & 5 & 1 & 3 \\
4 & 1 & 3 & 5 \\
\hline 4.09 & Table. $\quad(a, b, c, d) \bmod 6$. \\
\hline
\end{tabular}

Using the moduli 19 and 27 successively (noting that $c \geqq 3$ ) we have the following table:

\begin{tabular}{|rrrr|}
\hline$a$ & $b$ & $c$ & $d$ \\
\hline 6 & 11 & 7 & 7 \\
0 & 11 & 13 & 13 \\
0 & 9 & 5 & 11 \\
2 & 1 & 3 & 1 \\
14 & 7 & 9 & 13 \\
2 & 3 & 5 & 3 \\
8 & 9 & 17 & 3 \\
4 & 17 & 7 & 15 \\
10 & 7 & 3 & 17 \\
4 & 13 & 9 & 11 \\
\hline
\end{tabular}

4.10 Table. $(a, b, c, d) \bmod 18$.

Considering each of these cases mod 37 we have the following possibilities: $(a, b, c, d) \equiv(6,11,7,25),(2,1,3,1),(2,3,5,3)$ or $(4,17,7,33)$ $(\bmod (36,18,18,36))$. Using $\bmod 13$ we conclude $(a, b, c, d) \equiv(2,1,3,1)$ or $(2,3,5,3)(\bmod (36,36,18,36))$. Finally using $\bmod 5,(a, b, c, d) \equiv$ $(2,1,3,1)$ or $(2,3,5,3)(\bmod 36)$.

4.105 Lemma. $(a, b, c, d) \equiv(2,3,5,3)(\bmod 36)$. 
Proof. Suppose on the contrary that $(a, b, c, d) \equiv(2,1,3,1)$ $(\bmod 36)$. Since $d \geqq 2($ Lemma 4.083$)$ we have $7 \equiv 3^{\circ}(\bmod 25)$ so that $c \equiv 15(\bmod 20)$ and in fact $c \equiv 15(\bmod 30)$. Thus, using $\bmod 31$ we conclude that $b \equiv 8(\bmod 15)$, a contradiction.

\subsection{Lemma. $a=2$.}

Proof. Suppose on the contrary $a>2$. Since $b \equiv 3(\bmod 4)$ we conclude that $7^{3} \equiv 3^{c}(\bmod 25), c \equiv 5(\bmod 20)$ and in fact $c \equiv 5$ $(\bmod 180)$. Further $5^{2}+7^{b} \equiv 3^{5}+1(\bmod 31)$ so that $7^{b} \equiv 2(\bmod 31)$, $b \equiv 3(\bmod 15)$ and $b \equiv 3(\bmod 180)$. Examining cases $\bmod 61$ we conclude that $a \equiv 2, d \equiv 3(\bmod 30)$ so that $(a, b, c, d) \equiv(2,3,5,3)$ $(\bmod 180)$. Since $a, d \geqq 3,7^{b} \equiv 3^{c}(\bmod 125)$, so that $93 \equiv 3^{c}(\bmod 125)$, $c \equiv 65(\bmod 100)$. Applying these results $\bmod 101$ we conclude that $(a, b, c, d) \equiv(12,63,65,13)$ or $(22,3,65,8)(\bmod (25,100,100,25))$. In particular, $(a, b, c, d) \equiv(62,63,65,63)$ or $(122,3,65,183)(\bmod 300)$. Substituting these results mod 151 we have a contradiction. Hence $a=2$.

\subsection{Lemma. $a \neq 2$ (and hence $(a, b, c, d)$ does not exist).}

Proof. Suppose that $a=2$. Then $25+7^{b}=3^{c}+5^{d}$. (Recall that $(b, c, d) \equiv(3,5,3)(\bmod 36)$.) We consider two cases: $d=3$ and $d>3$.

4.1071 Case. $d=3$. Then $7^{b}=3^{c}+100$. Further (from Table $4.08), c \geqq 6$. Hence $7^{b} \equiv 100(\bmod 729)$ so that $b \equiv 84(\bmod 243)$ and in fact $b \equiv 327(\bmod 486)$. Since 3 is a primitive root $\bmod$ the prime 487 and since 7 has order $162(\bmod 487)$ and $327 \equiv 3(\bmod 162)$ we conclude immediately that $c \equiv 5(\bmod 486)$. We consider our equation modulo the prime 1459. Since 3 is a primitive root and the order of 7 is 243 , we have the following possibilities: $7^{84} \equiv 3^{5+4866}+100$ $(\bmod 1459), k=0,1,2$. Since $7^{84} \equiv 1016,3^{48} \equiv 339(\bmod 1459)$, in each case we have a contradiction. Hence $d>3$.

4.1072 Case. $d>3$. Since $7^{3} \equiv 3^{c}(\bmod 25)$ we conclude that $c \equiv 5(\bmod 20), \quad c \equiv 5(\bmod 180)$. Using $\bmod 31$ we conclude that $b \equiv 3(\bmod 15), b \equiv 3(\bmod 180)$. Thus, using $\bmod 11$ it immediately follows that $d \equiv 3(\bmod 5), d \equiv 3(\bmod 180)$. Further, since $25+$ $7^{b} \equiv 3^{c}(\bmod 125)$ we conclude that $118 \equiv 3^{c}(\bmod 125), c \equiv 5(\bmod 100)$. Examining possibilities $\bmod 101$ we have: $b \equiv 3(\bmod 100), d \equiv 3$ $(\bmod 25)$. Hence $368 \equiv 25+7^{3} \equiv 3^{c}(\bmod 625), \quad c \equiv 205(\bmod 500)$. We now consider our equation mod 251 . Since 5 has order 25 and 3,7 have order 125 we have: $25+7^{3+25 k} \equiv 3^{80}+5^{3}(\bmod 251), 0 \leqq$ 
$k \leqq 4$. Since $7^{25} \equiv 149,3^{80} \equiv 63(\bmod 251)$, in each case we deduce a contradiction. This completes the proof of the lemma and the theorem.

4.11 THEOREM. The only solutions to

$$
13^{a}+7^{b}=3^{c}+5^{d}
$$

are $(a, b, c, d)=(0,0,0,0),(0,1,1,1)$ and $(1,0,2,1)$.

Proof. Let $(a, b, c, d)$ be another solution. From [5], $a>0$. Using mod 3 we conclude that either $c=0$ and $d$ is even, or $c>0$ and $d$ is odd. In the former case $b>0$ so that using the moduli 8 and 7 successively we have a contradiction. Hence $c>0, d$ odd, so that using $\bmod 8$ we conclude $(a, b, c, d) \equiv(1,0,0,1)$ or $(0,1,1,1)$ $(\bmod 2)$. The theorem is a consequence of the following three lemmas.

\subsection{Lemma. $b>0$.}

Proof. Suppose that $b=0$. Then $13^{a}+1=3^{c}+5^{d},(a, c, d) \equiv$ $(1,0,1)(\bmod 2)$. Using $\bmod 5$ we conclude that $a \equiv 1, c \equiv 2(\bmod 4)$, so that, using $\bmod 16, d \equiv 1(\bmod 4)$. Thus, using $\bmod 13, c \equiv 2(\bmod 12)$. Then, using $\bmod 7$ we conclude that $d \equiv 1(\bmod 6), d \equiv 1(\bmod 12)$. We consider two cases: $d=1, d>1$.

4.1211 Case. $d=1$. Then $13^{a}=3^{c}+4$. Clearly $c \geqq 3$. Thus, using $\bmod 27, a \equiv 7(\bmod 9)$ so that $a \equiv 25(\bmod 36)$. This yields a contradiction $\bmod 37$. Hence $d>1$.

4.1212 Case. $d>1$. Then $13^{a}+1 \equiv 3^{c}(\bmod 25)$. Therefore, using $\bmod 25$ we have the following possibilities: $(a, c) \equiv(1,18)$, $(5,14),(9,10),(13,6)$ or $(17,2)(\bmod 20)$. Thus, using $\bmod 11$ we conclude that $(a, c, d) \equiv(1,38,49)(\bmod 60)$. This yields a contradiction $\bmod 61$. Hence $b>0$.

\subsection{Lemma. $(a, b, c, d) \equiv(0,1,1,1)(\bmod 2)$.}

Proof. Suppose the contrary. Then $(a, b, c, d) \equiv(1,0,0,1)(\bmod 2)$. Using $\bmod 7$ we conclude that $(c, d) \equiv(0,1)(\bmod 6)$ (and in particular $c \geqq 6)$. Using $\bmod 13$ we conclude that $b \equiv 4(\bmod 12), d \equiv 3(\bmod 4)$. Hence, using $\bmod 9, a \equiv 2(\bmod 3)$ so that $a \equiv 5(\bmod 6)$. Thus, using $\bmod 19,(a, b, c, d) \equiv(11,4,12,4)(\bmod (18,12,18,9))$ so that in fact $(a, b, c, d) \equiv(11,4,12,13)(\bmod (18,12,18,18))$. Therefore, using $\bmod 27$ we have $(a, b, c, d) \equiv(11,16,12,13)(\bmod (18,36,18,18))$ which yields a contradiction $\bmod 37$. Hence $(a, b, c, d) \equiv(0,1,1,1)(\bmod 2)$. 
4.123 Lemma. $\quad(a, b, c, d) \not \equiv(0,1,1,1)(\bmod 2)($ and hence $(a, b, c, d)$ does not exist).

Proof. Assume the contrary. Using mod 13 (and the fact that $c$ is odd) we have $(b, c, d) \equiv(7,3,1),(9,1,1)$ or $(5,1,3)(\bmod (12,6,4))$. Then, using $\bmod 7$ we have $(b, c, d) \equiv(9,1,1)$ or $(5,1,7)(\bmod (12,6,12))$. Thus, using $\bmod 5,(a, b, c, d) \equiv(0,9,1,1)$ or $(0,5,1,7)(\bmod (4,12,12,12))$. We consider two cases: $c=1$ and $c>1$.

4.1231 Case. $\quad c=1$. Then $13^{a}+7^{b}=3+5^{d}$. Clearly $d>1$. Using $\bmod 9$ we conclude that $(a, b, d) \equiv(8,9,1)$ or $(4,5,7)(\bmod 12)$. In either case, using $\bmod 25$ we find that $a \equiv 8(\bmod 20)$ so that $(a, b, d) \equiv(8,9,1)$ or $(28,5,7)(\bmod (60,12,12))$. Hence, using $\bmod 31$ we conclude that $(a, b) \equiv(8,45)(\bmod 60)$. This yields a contradiction $\bmod 11$. Hence $c>1$.

4.1232 Case. $c>1$. (Thus in fact $c \geqq 13$.) Using $\bmod 9$ we conclude that $(a, b, c, d) \equiv(4,9,1,1)$ or $(0,5,1,7)(\bmod 12)$. Then, using the moduli 19 and 27 successively we find $(a, b, c, d) \equiv(28,3,7,1)$, $(0,2,13,31)$ or $(12,8,7,7)(\bmod (36,9,18,36))$. In each case we have a contradiction mod 37 . Hence no other solution exists.

4.13 THEOREM. The only solutions to

$$
17^{a}+7^{b}=3^{c}+5^{d}
$$

are $(a, b, c, d)=(0,0,0,0)$ and $(0,1,1,1)$.

Proof. Let $(a, b, c, d)$ be another solution. From [5], $a>0$. We consider two cases: $c=0$ and $c>0$.

4.141 Case. $c=0$. Then $17^{a}+7^{b}=1+5^{d}$. Using $\bmod 3, a \equiv d$ $(\bmod 2)$. Hence, using $\bmod 16, b \equiv 0(\bmod 2), d \equiv 0(\bmod 4)$ and thus $a$ is even. Hence we have a contradiction mod 5 .

\begin{tabular}{|rrrr|}
\hline$a$ & $b$ & $c$ & $d$ \\
\hline 0 & 13 & 1 & 13 \\
0 & 9 & 5 & 1 \\
0 & 5 & 9 & 5 \\
0 & 1 & 13 & 9 \\
2 & 3 & 3 & 7 \\
2 & 15 & 7 & 11 \\
2 & 11 & 11 & 15 \\
2 & 7 & 15 & 3 \\
\hline
\end{tabular}

4.15 Table. $(a, b, c, d) \bmod (4,16,16,16)$. 
4.142 Case. $c>0$. Then $2^{a}+1 \equiv 2^{d}(\bmod 3)$ so that $(a, d) \equiv$ $(0,1)(\bmod 2)$. Hence, using the moduli 16,5 successively we conclude: $(a, b, c, d) \equiv(0,1,1,1)$ or $(2,3,3,3)(\bmod 4)$. Using $\bmod 17$ we have the possibilities listed in Table 4.15 .

In each of the above cases we have a contradiction mod 64. Hence there is no other solution.

\subsection{THEOREM. The solutions to}

$$
5^{a}+5^{b}=3^{c}+7^{d}
$$

are given in the following table:

\begin{tabular}{|cccc|}
\hline$a$ & $b$ & $c$ & $a$ \\
\hline 0 & 0 & 0 & 0 \\
1 & 1 & 1 & 1 \\
1 & 1 & 2 & 0 \\
1 & 3 & 4 & 2 \\
1 & 5 & 6 & 4 \\
3 & 1 & 4 & 2 \\
5 & 1 & 6 & 4 \\
2 & 2 & 0 & 2 \\
2 & 3 & 5 & 1 \\
\hline 4.18 & Table. &
\end{tabular}

Proof. Let $(a, b, c, d)$ be another solution. By [5], $a, b>0$. By Theorem 6.05, $a, b>1$. Immediately we conclude that $c \neq 1$ (using mod 25). The remainder of the proof is divided into five lemmas.

\subsection{Lemma. $c \geqq 2$.}

Proof. Assume the contrary. Then $c=0$ and $5^{a}+5^{b}=1+7^{d}$. If $a=2$ then $1+2^{1}+7^{d}=3^{3}+5^{b}$ and we have a contradiction by Theorem 6.01. Hence $a>2$ and by symmetry $b>2$. Thus $0 \equiv$ $1+7^{d}(\bmod 125)$ so that $d \equiv 10(\bmod 20)$. Further, using $\bmod 3, a$, $b$ are even. Therefore, using the moduli 7 and 9 successively we conclude that $a \equiv b \equiv 2(\bmod 6), d \equiv 2(\bmod 3)$ so that in fact $d \equiv 50$ $(\bmod 60)$. Thus we have a contradiction $\bmod 31$. Hence $c \geqq 2$.

\subsection{Lemma. $d>0$.}

Proof. Suppose $d=0 . \quad$ Then $5^{a}+5^{b}=3^{c}+1,3^{c} \equiv-1(\bmod 25)$, $c \equiv 10(\bmod 20)$. Using $\bmod 3, a$ and $b$ are odd. Thus, using $\bmod 7$, $(a, b, c) \equiv(1,1,2),(3,3,4),(3,5,0)$ or $(5,3,0)(\bmod 6)$. Thus $(a, b, c) \equiv$ 
$(1,1,50),(3,3,10),(3,5,30)$ or $(5,3,30)(\bmod (6,6,60))$. In each case we have a contradiction $\bmod 31$. Hence $d>0$.

4.183 Lemma. $(a, b, c, d) \equiv(3,3,5,1)(\bmod 60)$.

Proof. Using mod $3, a$ and $b$ are odd. Also, using $\bmod 8, c \equiv d$ $(\bmod 2)$. Since $0 \equiv 3^{c}+7^{d}(\bmod 25)$, we have $c \equiv 10+15 d(\bmod 20)$ so that $c \equiv 0(\bmod 5)$. Using these results and the moduli 7,9 successively we have the following table of possibilities:

\begin{tabular}{|llrl|}
\hline$a$ & $b$ & $c$ & $d$ \\
\hline 1 & 1 & 25 & 3 \\
1 & 3 & 10 & 2 \\
1 & 5 & 0 & 4 \\
3 & 1 & 10 & 2 \\
3 & 3 & 5 & 1 \\
3 & 5 & 20 & 0 \\
5 & 1 & 0 & 4 \\
5 & 3 & 20 & 0 \\
5 & 5 & 15 & 5 \\
\hline
\end{tabular}

4.19 Table. $(a, b, c, d) \bmod (6,6,30,6)$.

Hence, using $\bmod 31$ we have $(a, b, c, d) \equiv(1,1,25,21),(3,3,5,1)$ or $(5,5,15,11)(\bmod (6,6,30,30))$. (In particular $c$ and $d$ are odd.) Thus, using $\bmod 13$ we conclude $(a, b, c, d) \equiv(3,3,5,1)$ or $(11,11,15,11)$ $(\bmod (12,12,30,60))$. Thus, in either case, using $\bmod 16, c \equiv 1(\bmod 4)$. Hence, using $\bmod 5, d \equiv 1(\bmod 4)$ so that $(a, b, c, d) \equiv(3,3,5,1)$ $(\bmod (12,12,60,60))$. Thus, using $\bmod 11$ we conclude $(a, b, c, d) \equiv$ $(51,27,5,1),(27,51,5,1)$ or $(3,3,5,1)(\bmod 60)$. Therefore, using $\bmod 61,(a, b, c, d) \equiv(3,3,5,1)(\bmod 60)$.

\subsection{Lemma. $d>1$.}

Proof. Suppose $d=1$. Then $5^{a}+5^{b}=3^{c}+7$. By the previous lemma, $a, b \geqq 3$ so that $3^{c} \equiv-7(\bmod 125)$ and hence $c \equiv 5(\bmod 100)$ and in fact $c \equiv 5(\bmod 300)$. Therefore, using $\bmod 101$ we conclude that $(a, b, c) \equiv(3,3,5)(\bmod 300)$. We consider two cases: $a=3$ and $a>3$.

4.1941 Case. $a=3$. Then $118+5^{b}=3^{c}$. Clearly $b>3$. Thus, $118 \equiv 3^{c}(\bmod 625)$ so that $c \equiv 305(\bmod 500)$. We have an immediate contradiction mod 251. Hence $a>3$.

4.1942 Case. $a>3$. By symmetry, $b>3$ also. Hence $0 \equiv 3^{c}+7$ 
$(\bmod 625)$ so that $c \equiv 105(\bmod 500)$. Using $\bmod 251$ we again have a contradiction. Hence $d>1$.

4.195 Lemma. $d=1$. (Hence $(a, b, c, d)$ does not exist.)

Proof. Suppose $d>1$. Using $\bmod 71$ we conclude that $c \equiv 5$ $(\bmod 35), d \equiv 1(\bmod 70) . \quad$ In particular $(c, d) \equiv(5,1)(\bmod 42)$. Thus, using mod 49 we conclude that $(a, b) \equiv(3,39),(9,33),(15,27),(21,21)$, $(27,13),(33,9)$, or $(39,3)(\bmod 42)$. Applying these results $\bmod 43$ we conclude that $(a, b) \equiv(15,27)$ or $(27,15)(\bmod 42)$. Thus we have a contradiction mod 29. Hence no other solution exists.

While the previous theorems have not been easy, the following theorem, which would appear to be quite similar, is extremely easy.

4.20. THEOREM. The only solutions to

$$
5^{a}+7^{b}=3^{c}+7^{d}
$$

in nonnegative integers are $(a, b, c, d)=(0, t, 0, t), t$ arbitrary.

Proof. Let $(a, b, c, d)$ be another solution. Using $\bmod 3$ we conclude that $c=0$ and $a$ is even. Thus $a \geqq 2$ so that $7^{b} \equiv 1+7^{d}$ ( $\bmod 25)$, a contradiction.

4.22 THEOREM. The only nonnegative integral solutions to

$$
15^{a}+5^{b}=3^{c}+7^{d}
$$

are $(a, b, c, d)=(0,0,0,0),(1,0,2,1)$ and $(2,2,5,1)$.

Proof. Let $(a, b, c, d)$ be another solution. Clearly from [5], $a>0$. The theorem follows from several lemmas.

\subsection{LeMMA. $c>0$.}

Proof. Assume the contrary. Then $15^{a}+5^{b}=1+7^{d}$. Clearly $b, d>0$. Using mod 3 we find that $b$ is odd so that $\bmod 8$ we have an absurdity.

\subsection{Lemma. $b>0$.}

Proof. Suppose $b=0$. Then $15^{a}+1=3^{c}+7^{d}$. Clearly $a>1$, $c, d>0$. Thus, using $\bmod 7, c \equiv 2(\bmod 6)$. (In particular, $c \geqq 2$.) Hence, using $\bmod 9, d \equiv 0(\bmod 3)$. Thus, from $\bmod 15, d \equiv 1(\bmod 4)$ 
so that $d \equiv 9(\bmod 12)$. Also, $c \equiv 2(\bmod 4)$ so that $c \equiv 2(\bmod 12)$. Therefore, from $\bmod 13, a \equiv 4(\bmod 12)$. We consider two cases: $c=2$ and $c \geqq 3$.

4.2221 Case. $c=2$. Then $15^{a}=8+7^{d}$. Clearly $d>1,15^{a} \equiv 8$ $(\bmod 49), a \equiv 4(\bmod 7), a \equiv 4(\bmod 28), 7^{d} \equiv 12(\bmod 29)$, a contradiction. Hence $c>2$.

4.2222 Case. $\quad c \geqq 3$. Since $a \geqq 4$ we have $1 \equiv 7^{d}(\bmod 27), d \equiv 0$ $(\bmod 9), d \equiv 9(\bmod 36)$. Using $\bmod 19$ we conclude that $(a, c) \equiv(4,2)$, $(28,14)$ or $(16,26)(\bmod 36)$. In each case we consider our equation $\bmod 73$ and deduce a contradiction. Hence $b \geqq 1$.

\subsection{LeMMA. $d>0$.}

Proof. Suppose $d=0$. Then $15^{a}+5^{b}=3^{c}+1$ (and $a b c>0$ ). Using $\bmod 3, b$ is even. Using $\bmod 5, c \equiv 2(\bmod 4)$. Hence, using $\bmod 8, a$ is even (and $a \geqq 2)$. Thus, using $\bmod 9, b \equiv 0(\bmod 6)$. Hence, using $\bmod 7, c \equiv 0(\bmod 6)$ and in fact $c \equiv 6(\bmod 12)$. Combining the above results and using mod 16 we conclude that $b \equiv 2(\bmod 4)$. Thus, from $\bmod 13, a \equiv 4(\bmod 12)$. Hence $5^{b} \equiv 1$ $(\bmod 27)$ so that $b \equiv 0(\bmod 18)$. Considering all possibilities $\bmod 19$ we deduce a contradiction. Hence $a b c d>0$.

\subsection{Lemma. $(a, b, c, d) \equiv(2,2,5,1)(\bmod 60)$.}

Proof. Using $\bmod 3, b$ is even (and so $b \geqq 2$ ). Using $\bmod 5$, $d \equiv c(\bmod 2)$. Hence, using $\bmod 8, a$ is even $(a \geqq 2)$. Using the moduli 7 and 9 successively we have the following possibilities: $(b, c, d) \equiv(0,2,0),(2,5,1)$ or $(4,1,5)(\bmod 6)$. Further, $0 \equiv 3^{c}+7^{d} \equiv$ $3^{c}+3^{15 d}(\bmod 25)$ so that $c \equiv 10+15 d(\bmod 20)$ and, in particular, $c \equiv 0(\bmod 5)$. Hence, using $\bmod 31$ we have $(a, b, c, d) \equiv(2,0,20,6)$ or $(2,2,5,1)(\bmod (10,6,30,30))$. Thus, using $\bmod 11$, we have $(a, b, c, d) \equiv(2,2,5,1)(\bmod (10,30,30,30))$. Using $\bmod 61$ we conclude that $a \equiv 2(\bmod 15), d \equiv 1(\bmod 60)$. Hence $a \equiv 2(\bmod 30)$. Therefore, using $\bmod 5, c \equiv 1(\bmod 4)$ so that $c \equiv 5(\bmod 60)$. Thus, using $\bmod 16, b \equiv 2(\bmod 4), b \equiv 2(\bmod 60)$. Hence, using $\bmod 13, a \equiv 2$ $(\bmod 12)$ and in fact $(a, b, c, d) \equiv(2,2,5,1)(\bmod 60)$ as asserted.

\subsection{LEMMA. $d>1$.}

Proof. Assume the contrary. Then $15^{a}+5^{b}=3^{c}+7$. Since $(a, b, c) \equiv(2,2,5)(\bmod 60)$, clearly $c \geqq 65$. We consider two cases: $a=2$ and $a>2$. 
4.2251 Case. $a=2$. Then $218+5^{b}=3^{c}$, so that $218+5^{b} \equiv 0$ $(\bmod 2187)$. Hence $b \equiv 650(\bmod 1458)$. Using $\bmod 487$ we easily conclude that $c \equiv 5(\bmod 486)$. Thus, using $\bmod 1459$ we have a contradiction. Hence $a>2$.

4.2252 Case. $a>2$. Recall that $c \geqq 5$ so that $5^{b} \equiv 7(\bmod 27)$, $b \equiv 14(\bmod 18)$. Thus we have a contradiction $\bmod 19$.

4.226 Lemma. $d=1$ (so that $(a, b, c, d)$ does not exist).

Proof. Suppose instead that $d>1$. Using $\bmod 43$ we have the following table:

\begin{tabular}{|rrr|}
\hline$a$ & $b$ & $c$ \\
\hline 2 & 2 & 5 \\
2 & 38 & 29 \\
8 & 8 & 11 \\
8 & 20 & 23 \\
8 & 32 & 17 \\
38 & 14 & 17 \\
38 & 20 & 35 \\
20 & 8 & 41 \\
20 & 26 & 11 \\
\hline 4.24 & Table. & $(a, b, c) \bmod 42$.
\end{tabular}

(Recall that $d \equiv 1(\bmod 60)$ ) $)$ Thus, using $\bmod 49$ we conclude that $(a, b, c) \equiv(8,8,11)$ or $(20,8,41)(\bmod 42)$. Hence, using $\bmod 29$, $(a, b, c, d) \equiv(22,22,25,5)(\bmod 28)$. Thus, combining all of our results we have a contradiction when our equation is considered modulo 71.

4.25 Theorem. The only solutions to

$$
15^{a}+3^{b}=5^{c}+7^{d}
$$

in nonnegative integers are $(a, b, c, d)=(0,0,0,0)$ or $(2,5,3,3)$.

Proof. By [5], $a>0$. Thus, using $\bmod 3, b \neq 0$ and $c$ is odd. Then $3^{b} \equiv 2^{d}(\bmod 5)$ so that $b \equiv d(\bmod 2)$. Hence, using $\bmod 8$ we conclude that $a$ is even and $b$ and $d$ are odd. Thus, using mod 7, $(b, c) \equiv(5,3)(\bmod 6), b \geqq 5$. Thus $0 \equiv 5^{3}+7^{d}(\bmod 9)$ so that $d \equiv 0$ $(\bmod 3), d \equiv 3(\bmod 6)$. Using $\bmod 13$ we conclude $(a, b, c, d) \equiv$ $(0,5,9,3),(2,5,9,9)$ or $(2,5,3,3)(\bmod (12,6,12,12))$. Thus, using $\bmod 16,(a, b, c, d) \equiv(0,11,9,3),(2,11,9,9) \quad$ or $(2,5,3,3)(\bmod 12)$. 
Using mod 19 and combining results we have the following table:

\begin{tabular}{rrrr|}
\hline$a$ & $b$ & $c$ & $d$ \\
\hline 24 & 11 & 9 & 3 \\
2 & 23 & 21 & 9 \\
14 & 11 & 33 & 9 \\
2 & 5 & 3 & 3 \\
14 & 11 & 15 & 3 \\
\hline
\end{tabular}

4.27 Table. $(a, b, c, d) \bmod (36,36,36,12)$.

The remainder of the proof is divided into two lemmas.

4.271 Lemma. $a=2$ and $(b, c, d) \equiv(5,3,3)(\bmod 36)$.

Proof. Assume $a>2$. Then $0 \equiv 5^{c}+7^{d}(\bmod 27)$. Thus, $c \equiv$ $14 d-9(\bmod 18)$. Combining results, $(c, d) \equiv(9,27),(21,33),(33,21)$, $(3,15)$ or $(15,3)(\bmod 36)$. In each case we have a contradiction mod 37. Hence $a=2$. Thus, from Table $4.27,(b, c, d) \equiv(23,21,9)$ or $(5,3,3)(\bmod (36,36,12))$. In either case, $9 \equiv 5^{3}+7^{d}(\bmod 27)$ so that $d \equiv 3(\bmod 9)$. Thus, in the former case, we have a contradiction $\bmod 37$. Hence $(b, c, d) \equiv(5,3,3)(\bmod 36)$.

\subsection{Lemma. $c>3$.}

Proof. Assume the contrary. Then $c=3$ and $100+3^{b}=7^{d}$, $b \geqq 6$. From Lemma 4.107, case 4.1071 , we immediately have a contradiction.

4.273 Lemma. $c \leqq 3$ (and hence $(a, b, c, d)$ does not exist).

Proof. Assume the contrary. (Recall that $a=2,(b, c, d) \equiv$ $(5,3,3)(\bmod 36)$.$) \quad Since 3^{b} \equiv 18(\bmod 25), \quad b \equiv 5(\bmod 20), \quad b \equiv 5$ $(\bmod 180) . T h u s$, using $\bmod 31, d \equiv 3(\bmod 15), d \equiv 3(\bmod 180)$. Immediately then, using $\bmod 11, c \equiv 3(\bmod 5), c \equiv 3(\bmod 180)$. Since $3^{b} \equiv 118(\bmod 125), \quad b \equiv 5(\bmod 100)$. Thus, using $\bmod 101$, $c \equiv 3(\bmod 25), \quad d \equiv 3(\bmod 100)$ and in fact $(b, c, d) \equiv(5,3,3)$ $(\bmod 900) . \quad T h u s \quad 3^{b} \equiv 118(\bmod 625), \quad b \equiv 305(\bmod 500), \quad b \equiv 1805$ $(\bmod 4500)$. Hence, using $\bmod 251, d \equiv 53(\bmod 125)$ so that $d \equiv 1803$ $(\bmod 4500)$. Finally, we consider the prime $751 . \quad(3,5$, and 7 have orders 750,375 and 250 respectively.) Thus $225+3^{305} \equiv$ $5^{3+75 k}+7^{53}(\bmod 751), 0 \leqq k \leqq 4$. Since $3^{305} \equiv 630,5^{75} \equiv 569$ and $7^{53} \equiv$ 27 , in each case we have a contradiction. Hence no other solution exists. 
5. Theorems concerning equations of the general form $p^{a}+q^{b}=r^{c}+s^{d}$. Many exponential diophantine equations (e.g., $13^{a}+5^{b}=3^{c}+7^{d}, 19^{a}+5^{b}=3^{c}+7^{d}$ ) can be shown to have only the trivial solution. In such cases proofs are easily generalized to include infinite classes of equations.

\subsection{ThEOREM. The equation}

$$
n^{a}+3^{b}=5^{c}+7^{d}
$$

has no nontrivial solution if the three conditions $n \equiv 1(\bmod 6)$, $n \not \equiv 0(\bmod 5), n \neq \equiv \pm 1(\bmod 8)$ all hold.

Proof. Suppose $(a, b, c, d)$ is another solution. Using $\bmod 3$ we conclude that $b=0$ and $c$ is even. Hence, using $\bmod 8, a$ and $d$ are even. Since $b=0$ we conclude $c \neq 0$. Hence we have a contradiction $\bmod 5$.

We have two immediate corollaries:

5.021 COROLlaRY. Equation 5.02 has no nontrivial solutions for the primes $n=13,19,37,43$, and 61 .

5.022 COROLlary. Equation 5.02 has no nontrivial solutions for $n \equiv 13(\bmod 120)$ (and hence for infinitely many prime $n)$.

5.03 THEOREM. The equation

$$
n^{a}+5^{b}=3^{c}+7^{d}
$$

has no nontrivial solution if $n \equiv 1(\bmod 6), n \equiv \pm 1(\bmod 5)$ and $n \not \equiv \pm 1(\bmod 8)$.

Proof. Let $(a, b, c, d)$ be another solution. Using mod 3 we conclude that $c=0$ and $b$ is even. Hence, using $\bmod 8, a$ and $d$ are even. Thus, using mod 5 we have a contradiction.

Therefore we have:

5.041 Corollary. Equation 5.04 has no nontrivial solution for the primes $n=19,61,109,139$, and 181 .

5.042 COROLlary. Equation 5.04 has no nontrivial solution if $n \equiv 19(\bmod 120)$.

5.05 THEOREM. Equation 5.04 has no nontrivial solution if 
$n \equiv 1(\bmod 6), n \equiv \pm 2(\bmod 5)$ and $n \neq \equiv \pm 1(\bmod 8)$

Proof. Let $(a, b, c, d)$ be another solution. Using $\bmod 3$ we conclude that $c=0$ and $b$ is even. Therefore, using $\bmod 8, a$ and $d$ are even. Thus we have a contradiction $\bmod 5$.

5.051 CoRollary. Equation 5.04 has no nontrivial solution for the primes $n=13,37,43,67,133$ and 157.

5.052 COROLlaRY. Equation 5.04 has no nontrivial solution if $n \equiv 13(\bmod 120)$.

5.06 THEOREM. Equation 5.04 has no nontrivial solution if $n \equiv 1$ or $13(\bmod 42)$ and $n \neq \equiv \pm 1(\bmod 8)$.

Proof. Let $(a, b, c, d)$ be another solution. Using $\bmod 3$ we conclude that $c=0$ and $b$ is even. Thus, using $\bmod 8, a$ and $d$ are even. Since $c=0, d>0$ and we have a contradiction $\bmod 7$.

5.061 COROLlary. Equation 5.04 has only the trivial solution for the primes $n=211,307,349,379,421,547,643$ and 757.

5.062 COROLLARY. Equation 5.04 has no nontrivial solution for $n \equiv 13(\bmod 168)$.

5.07 THEOREM. Equation 5.04 has no nontrivial solution if $n \equiv 17(\bmod 120)$.

Proof. Let $(a, b, c, d)$ be another solution. Using mod 3 we find that either $c=0$ and $a$ and $b$ are even or $c>0$ and $a$ and $b$ are odd. Thus, using $\bmod 8, c=0$ and is even. Therefore $b>0$ and we deduce a contradiction using $\bmod 5$.

The following two theorems are generalizations of [5].

5.08 TheOREM. Let $n=3 \cdot 5^{2} \cdot 7 \cdot 11 \cdot m$ where $m \equiv-1(\bmod 8)$. Then the equation

$$
n^{a}+7^{b}=3^{c}+5^{d}
$$

has only the solutions $(a, b, c, d)=(0,0,0,0),(0,1,1,1)$.

Proof. Suppose $(a, b, c, d)$ is another solution of (5.09). By [5], $a \neq 0$. Hence, using $\bmod 3, c>0$ and $d$ is even. Thus, using $\bmod 8$, $b$ and $c$ are even. We consider two cases: $b=0$ and $b>0$. 
5.091 CASE. $b=0$. Then $n^{a}+1=3^{c}+5^{d}$. Clearly $d>0$ so that in fact $d \geqq 2$. Hence $1 \equiv 3^{c}(\bmod 25)$ so that $c \equiv 0(\bmod 20)$. Thus we have a contradiction $\bmod 11$. Hence $b>0$.

5.092 CASE. $b>0$. Then $0 \equiv 3^{c}+5^{d}(\bmod 7)$, a contradiction.

5.10 THEOREM. Let $n \equiv 1\left(\bmod 2^{4} \cdot 3^{2} \cdot 5^{2} \cdot 7 \cdot 13\right)$. Then the only solutions of (5.09) are $(a, b, c, d)=(0,0,0,0)$ and $(0,1,1,1)$.

Proof. Suppose $(a, b, c, d)$ is another solution. Using $\bmod 3$ we conclude $d>0$. Hence $7^{b} \equiv 3^{c}+4(\bmod 20)$ so that $b \equiv c \equiv 1(\bmod 4)$. Thus, using $\bmod 16, d \equiv 1(\bmod 4)$. Therefore, using $\bmod 13, c \equiv 1$ $(\bmod 3)$ and $b \equiv 1(\bmod 12)$. Hence $c \equiv 1(\bmod 6), 5^{d} \equiv 5(\bmod 7)$ and $d \equiv 1(\bmod 6)$. Using $\bmod 25$ we conclude that $c>1$. Finally we have a contradiction $\bmod 9$.

We present the following easy general theorems without proof.

5.11 THEOREM. Let $p, q, r$ be distinct primes. Suppose $p \equiv$ $q \equiv 1(\bmod r), p \equiv 1(\bmod q)$. Then the only solutions of

$$
p^{a}+q^{b}=p^{c}+r^{d}
$$

are $(a, b, c, d)=(t, 0, t, 0)$, where $t$ is any integer.

5.121 Corollary. If

$$
43^{a}+7^{b}=43^{c}+3^{d}
$$

then $a=c, b=d=0$.

5.14 THEOREM. Let $p$ be prime. Then the only solutions of

$$
p^{a}+p^{b}=p^{c}+p^{d}
$$

are $(a, b, c, d)=(s, t, s, t)$ and $(a, b, c, d)=(s, t, t, s)$ where $s, t$ are arbitrary integers.

5.16 TheOREM. Let $p, q$ be distinct odd primes such that $q \equiv 1$ $(\bmod p)$. Then the equation

$$
p^{a}+p^{b}=q^{c}+q^{d}
$$

has only the trivial solution.

5.18 REMARK. The essential hypotheses in Theorems 5.11, 5.14 and 5.16 are the congruence relations. The primality of the bases plays no rôle in the arguments. 
6. The equations $1+2^{a}+7^{b}=3^{c}+5^{d}, 3^{a}+7^{b}=3^{c}+5^{d}+2$. The equations of this section have numerous solutions. The proofs given here are self-contained in the sense that they do not depend on results from any other section. On the other hand, the results established here are used in $\S \S 3$ and 4.

\subsection{THEOREM. The only solutions of}

$$
1+2^{a}+7^{b}=3^{c}+5^{d}
$$

in nonnegative integers are given in Table 6.03.

\begin{tabular}{|cccc|}
\hline$a$ & $b$ & $c$ & $d$ \\
\hline 1 & 0 & 1 & 0 \\
1 & 1 & 2 & 0 \\
1 & 2 & 3 & 2 \\
2 & 0 & 0 & 1 \\
3 & 0 & 2 & 0 \\
5 & 0 & 2 & 2 \\
5 & 2 & 4 & 0 \\
\hline
\end{tabular}

6.03 Table. The solutions of (6.02).

Proof. Suppose $(a, b, c, d)$ is another solution.

6.031 Lemma. $b>0$.

Proof. Suppose $b=0$ so that $2+2^{a}=3^{c}+5^{d}$. We consider several cases.

6.0311 Case. $d=0$. Then $1+2^{a}=3^{c}$. Clearly $c \geqq 3$, so that using $\bmod 27, a \equiv 9(\bmod 18)$. This yields a contradiction $\bmod 19$.

6.0312 Case. $d=1$. Then $2^{a}=3^{c}+3$. Clearly $c>0$ so that using $\bmod 3$ we have a contradiction.

6.0313 Case. $c=0, d>1$. Then $1+2^{a}=5^{d}$. Clearly $a \geqq 3$. Using $\bmod 8$ we conclude that $d$ is even. But then we have a contradiction $\bmod 3$.

6.0314 Case. $c=1, d>1$. Then $2^{a}=1+5^{d}$. Clearly $a \geqq 3$ so that we have a contradiction $\bmod 8$.

6.0315 Case. $\quad c, d \geqq 2$. Then $2+2^{a}=3^{c}+5^{d}$. Clearly $a \geqq 6$. Using $\bmod 8$ we see that $c$ and $d$ are even. Hence, using $\bmod 3, a$ 
is odd. Therefore, using $\bmod 5, a \equiv 1, c \equiv 2(\bmod 4)$, so that, using $\bmod 16, d \equiv 2(\bmod 4)$. Hence (using $\bmod 13), a \equiv 5(\bmod 12), c \equiv 2$ $(\bmod 3)$ so that in fact $c \equiv 2(\bmod 12)$. Using $\bmod 9$ we conclude that $d \equiv 2(\bmod 12)$. Using $\bmod 19$ we conclude that $(a, c, d) \equiv$ $(5,2,2),(5,8,14)$ or $(17,8,2)(\bmod (36,18,36))$. Thus, using $\bmod 37$ we have $(a, c, d) \equiv(5,2,2)(\bmod 36)$. Therefore, using $\bmod 73, d \equiv 2$ $(\bmod 72)$ so that using $\bmod 32, c \equiv 2(\bmod 8)$. Hence, using $\bmod 64$ we find that $(c, d) \equiv(2,10)$ or $(10,2)(\bmod 16)$. Finally, we have a contradiction $\bmod 17$.

\subsection{Lemma. $c>0$.}

Proof. Suppose $c=0$. Then $2^{a}+7^{b}=5^{d}$. Using $\bmod 3$ we find that $d$ is odd and $a$ is even. Hence, using $\bmod 8, a=2$. Thus we have a contradiction $\bmod 7$.

\subsection{LEMMA. $a>0$.}

Proof. Suppose $a=0$. Then $2+7^{b}=3^{c}+5^{d}$. This reduces to an absurdity modulo 3 .

\subsection{Lemma. $d>0$.}

Proof. Suppose $d=0$. Then $2^{a}+7^{b}=3^{c}$. Clearly $c \geqq 3$. Also, using $\bmod 3, a$ is odd. We consider several cases:

6.0341 Case. $a=1$. Then $2+7^{b}=3^{c}, c \geqq 3$. Using $\bmod 7$, we find $c \equiv 2(\bmod 6)$. Further, using $\bmod 27, b \equiv 4(\bmod 9)$. Thus we have a contradiction mod 37.

6.0342 Case. $a=3$. Then $8+7^{b}=3^{c}$. Using $\bmod 7, c \equiv 0$ $(\bmod 6)$. Also, using $\bmod 9, b \equiv 0(\bmod 3)$. This yields a contradiction $\bmod 19$.

6.0343 Case. $a=5$. Then $32+7^{b}=3^{c}$. Using $\bmod 8$ we conclude that $b, c$ are even. Also, clearly $b \geqq 4$. Thus $c \geqq 5,7^{b} \equiv-32 \equiv$ $(-2)^{5}(\bmod 243)$. From this it follows easily that $b \equiv 29(\bmod 81)$. Hence in fact $b \equiv 110(\bmod 162)$. Thus using $\bmod 163$ we have $c \equiv 95$ $(\bmod 162)$, so that $c$ is odd, a contradiction.

6.0344 Case. $a \geqq 7$. Then $2^{a}+7^{b}=3^{c}, a \geqq 7$. Using $\bmod 16$ we find that $b$ is even and $c \equiv 0(\bmod 4)$. Hence using $\bmod 5, a \equiv 1$, $b \equiv 2(\bmod 4)$. Thus, using $\bmod 64$ we deduce that $(b, c) \equiv(2,12)$ or $(6,4)(\bmod (8,16))$. Applying these results $\bmod 17$ we have 


$$
(a, b, c) \equiv(1,10,12)(\bmod (8,16,16)) .
$$

Further, using $\bmod 13,(a, b, c) \equiv(1,6,0),(5,2,1)$ or $(9,10,2)(\bmod$ $(12,12,3))$. Thus, using $\bmod 7,(a, b, c) \equiv(5,2,1)(\bmod (12,12,3))$, so that in fact, from $(6.0345),(a, b, c) \equiv(17,26,28)(\bmod (24,48,48))$. Finally, we have a contradiction $\bmod 97$.

\subsection{Lemma. $a>1$.}

Proof. Suppose $a=1$. Then $3+7^{b}=3^{c}+5^{d}$. Thus $c \geqq 2$. Using $\bmod 3$ we conclude that $d$ is even. Using $\bmod 8, b \neq c(\bmod 2)$. Using $\bmod 7$ and $\bmod 9$ we conclude $(b, c, d) \equiv(3,0,4),(1,2,0)$ or $(2,3,2)(\bmod 6)$. Using $\bmod 105$ we find $(b, c, d) \equiv(3,0,4),(7,8,0)$ or $(2,3,2)(\bmod (12,12,6))$. Applying these results $\bmod 13$ we conclude $(b, c, d) \equiv(2,3,2)(\bmod 12)$, so that, using $\bmod 37,(b, c, d) \equiv$ $(2,3,2)(\bmod 36)$. Further, using $\bmod 109$ we find that $(b, c, d) \equiv$ $(2,3,2)(\bmod 27)$. If $c>3$ we have a contradiction $\bmod 81$. Hence $c=3, d>2,7^{b} \equiv 24(\bmod 125), b \equiv 6(\bmod 20)$. This yields a contradiction $\bmod 11$.

6.0347 Lemma. $a \equiv 1, b \equiv c \equiv d \equiv 0(\bmod 2)$.

Proof. Using $\bmod 3, a \equiv 1, d \equiv 0(\bmod 2)$. Thus using $\bmod 8$, $b \equiv c \equiv 0(\bmod 2)$.

6.0348 Lemma. $a<3$. (This eliminates all remaining cases and completes the proof of the theorem.)

Proof. Suppose $a \geqq 3$. If $a=3$ we immediately have a contradiction $\bmod 5$. Hence $a \geqq 5$. Using $\bmod 80$ we conclude $(a, b, c, d) \equiv$ $(1,0,2,2)(\bmod 4)$. Using $\bmod 13$ therefore, $(a, b, c, d) \equiv(5,0,2,2)$ or $(9,4,10,2)(\bmod (12,12,12,4))$. Thus we have a contradiction $\bmod 7$.

The following theorem concerns a five term equation with infinitely many solutions.

\subsection{THEOREM. The only solutions of}

$$
3^{a}+7^{b}=3^{c}+5^{d}+2
$$

in nonnegative integers are given in Table 6.07. In this table, $t$ denotes an arbitrary nonnegative integer.

Proof. Let $(a, b, c, d)$ be another solution. 


\begin{tabular}{|llll|}
\hline$a$ & $b$ & $c$ & $d$ \\
\hline$t$ & 1 & $t$ & 1 \\
1 & 0 & 0 & 0 \\
2 & 0 & 1 & 1 \\
3 & 0 & 0 & 2 \\
3 & 3 & 5 & 3 \\
4 & 2 & 1 & 3 \\
6 & 4 & 1 & 5 \\
\hline
\end{tabular}

6.07 Table. The solutions of (6.06).

\subsection{LeMMA. $a, d>0$.}

Proof. Either $a=0$ or $a>0$. Suppose $a=0$. Using $\bmod 3$ we conclude $c=0, d$ odd. Hence in particular, $7^{b} \equiv 2(\bmod 25)$, a contradiction. Hence $a>0$. Next suppose $a \neq 0, d=0$. Using $\bmod 3$ we conclude that $c=0$, a contradiction.

\subsection{LEMMA. $d>1$.}

Proof. Suppose $d=1$. Then $3^{a}+7^{b}=3^{c}+7$. If $b$ were 0 we would have a contradiction $\bmod 9$. Hence $b \geqq 2$. Using $\bmod 7$ we conclude $a \equiv c(\bmod 6)$ so that $7^{b} \equiv 7(\bmod 13), b \equiv 1(\bmod 12)$. Hence $b \equiv 1(\bmod 6)$. Now $3^{a} \equiv 3^{c}+7(\bmod 49)$ so that $a \neq \equiv c$ (mod 42). This contradicts the fact that $3^{a} \equiv 3^{c}(\bmod 43)$. (For, 3 is a primitive root for each of the moduli 49,43 . Also, $b \equiv 1$ $(\bmod 6)$ and $\left.7^{6} \equiv 1(\bmod 43).\right)$

\subsection{LEMMA. $b>0$.}

Proof. Suppose $b=0,3^{a}=3^{c}+5^{d}+1$. We consider two cases: $c=0, c>0$.

6.0731 Case. $c=0$. Then $3^{a}=5^{d}+2$. Using $\bmod 3, d$ is even. Hence $3^{a}>27$. Using the moduli 9,7 successively we have: $d \equiv 2$, $a \equiv 3(\bmod 6)$. Therefore, using $\bmod 19$ we conclude $a \equiv 3(\bmod 18)$, $d \equiv 2(\bmod 9)$. Since $a \geqq 4$ we have $5^{d} \equiv 79(\bmod 81), d \equiv 20(\bmod 54)$. Thus we have a contradiction mod 109 .

6.0732 Case. $c>0$. Using mod 3, $d$ is odd. Also, by Lemma 2, $d \geqq 3$. Applying mod 8 we find that $a$ is even and $c$ is odd. By inspection, $a \geqq 4$. Furthermore, using $\bmod 5, a \equiv 2, c \equiv 1(\bmod 4)$, so that, using $\bmod 16, d \equiv 1(\bmod 4)$. Suppose $c=1,3^{a}=5^{d}+4$. Using $\bmod 27$ we conclude that $d \equiv 13(\bmod 18)$. Hence using $\bmod 7$, $a \equiv 2(\bmod 6)$, and thus we have a contradiction $\bmod 19$. Therefore, 
$c>1$ and in fact $c \geqq 5$. Using $\bmod 9$ we have $d \equiv 3(\bmod 6)$, which yields a contradiction $\bmod 7$.

\subsection{Lemma. $c>0$.}

Proof. Suppose $c=0,3^{a}+7^{b}=5^{d}+3$. Using mod 3 we conclude that $d$ is even. Thus, using $\bmod 8, a$ is odd and $b$ is even. Hence, using $\bmod 5, a \equiv 3(\bmod 4)$. Therefore, using $\bmod 7, a \equiv 5, d \equiv 4$ $(\bmod 6)$, so that using $\bmod 9, b \equiv 4(\bmod 6)($ since $b$ is even). Hence we have a contradiction $\bmod 13$.

\subsection{LEMMA. $a>1$.}

Proof. Suppose the contrary. Then $a=1,1+7^{b}=3^{c}+5^{d}$. Using $\bmod 20, b \equiv c \equiv 1(\bmod 4) . \quad H e n c e$, using $\bmod 16, d \equiv 1(\bmod 4)$. Examining possibilities $\bmod 13$ we have $b \equiv 1(\bmod 12), c \equiv 1(\bmod 3)$. Therefore $c \equiv 1(\bmod 6), 5 \equiv 5^{d}(\bmod 7)$ and hence $d \equiv 1(\bmod 6)$. Thus, if $c>1$ we have a contradiction $\bmod 9$. Further, if $c=1$, $d>1$ we have a contradiction $\bmod 25$.

\subsection{LemMa. $a>2$.}

Proof. Suppose $a=2$. Then $7+7^{b}=3^{c}+5^{d}$. Using mod 3, $d$ is odd. Hence using the moduli 7,8 successively we conclude that $c$ is even, $b$ is odd. Thus, using $\bmod 5, b \equiv 1, c \equiv 2(\bmod 4)$. Using $\bmod 13,(b, c, d) \equiv(1,2,1)(\bmod (12,3,4))$, and, in fact $c \equiv 2(\bmod 12)$. Hence, using $\bmod 7, d \equiv 1(\bmod 6)$. Therefore, using $\bmod 49$ we conclude $(c, d) \equiv(2,19),(8,25),(14,31), \quad(20,37),(26,1),(32,7)$ or $(38,13)(\bmod 42)$. Consideration of our equation $\bmod 43$ in each of these cases yields a contradiction.

\subsection{LemMa. $c>1$.}

Proof. Suppose $c=1,3^{a}+7^{b}=5^{d}+5$. Using $\bmod 3, d$ is odd. Using $\bmod 8, a \equiv b(\bmod 2)$. Using $\bmod 7,(a, d) \equiv(1,1),(4,3)$ or $(0,5)(\bmod 6) . \quad$ Thus, using $\bmod 9,(b, d) \equiv(0,1),(2,3)$ or $(1,5)$ $(\bmod (3,6))$. Combining these results we conclude that $(a, b, d) \equiv$ $(1,3,1),(4,2,3)$ or $(0,4,5)(\bmod 6)$. Applying $\bmod 13$ we have: $(a, b, d) \equiv(1,2,3)$ or $(0,4,1)(\bmod (3,12,4))$ so that in fact $(a, b, d) \equiv$ $(4,2,3)$ or $(0,4,5)(\bmod (6,12,12))$. Using $\bmod 5,(a, b, d) \equiv(4,2,3)$ or $(6,4,5)(\bmod 12)$. Applying these results $\bmod 37$ we conclude that $(a, b, d) \equiv(4,2,3)$ or $(6,4,5)(\bmod 36)$. Hence, using $\bmod 109$,

$$
(a, b, d) \equiv(4,2,3) \quad \text { or }(6,4,5)(\bmod 108) \text {. }
$$


Consideration of our equation mod 163 then yields $(a, b, d) \equiv(4,2,3)$ or $(6,4,5)(\bmod (162,162,54))$. We consider three cases: $a>6, a=4$, $a=6$.

6.0781 Case. $a>6$. Using $\bmod 243$ we have $(a, b, d) \equiv(4,2,111)$ or $(6,4,5)(\bmod 162)$. Applying $\bmod 487$ we find $(a, b, d) \equiv(4,2,111)$ or $(6,4,5)(\bmod (486,162,162))$. Using $\bmod 729$ we conclude $(a, b, d) \equiv$ $(4,2,435),(4,326,273),(4,164,111),(6,4,5),(6,328,329)$ or $(6,166,167)$ $(\bmod 486)$. Consideration of our equation in each of these cases $\bmod 1459$ yields $(a, b, d) \equiv(6,4,5)(\bmod (1458,486,486))$. Therefore, using $\bmod 2187$ we conclude $(a, b, d) \equiv(6,4,977),(6,247,491)$ or $(6,490,5)(\bmod (1458,729,1458))$. Since $(a, b, d) \equiv(6,4,5)(\bmod 36)$ (from (6.08) and the fact that $a \equiv 0(\bmod 6))$ we conclude $(a, b, d) \equiv$ $(6,4,977),(6,976,491)$, or $(6,1948,5)(\bmod 2916)$. In each case we have a contradiction $\bmod 2917$. Hence $a \leqq 6$ so that $a=4$ or $a=6$.

6.0782 Case. $a=4$. Then $76+7^{b}=5^{d}$. Hence $d \geqq 4,7^{b} \equiv 549$ $(\bmod 625)$ so that $b \equiv 22(\bmod 100)$. Thus, using $\bmod 101$ we have a contradiction.

6.0783 Case. $\quad a=6 . \quad 724+7^{b}=5^{d}$. Hence $d>5, \quad 7^{b} \equiv 14901$ $(\bmod 15625) . \quad$ Thus $b \equiv 2004(\bmod 2500)$ so that $b \equiv 27004(\bmod 67500)$. (For, from $(6.08), b \equiv 4(\bmod 108)$ ) $) \quad$ Using $\bmod 101, d \equiv 5(\bmod 25)$. Hence $d \equiv 5(\bmod 2700)$. Therefore, using $\bmod 11251$ we have a contradiction. Thus $e>1$.

\subsection{Lemma. $b>1$.}

Proof. Suppose $b=1, \quad 3^{a}+5=3^{c}+5^{d}$. Using $\bmod 9$, we conclude $d \equiv 1(\bmod 6)$. Hence $3^{a}+5 \equiv 3^{c}(\bmod 25)$. Thus (since 3 is a primitive root $\bmod 25), a \neq \equiv c(\bmod 20)$. Also $a \not \equiv c(\bmod 10)($ or we would have $\left.2 \cdot 3^{c} \equiv 5(\bmod 25)\right)$. Since $5^{d} \equiv 5(\bmod 31)$ and 3 is a primitive root mod 31 we have a contradiction.

\subsection{Lemma. $c>2$.}

Proof. Suppose on the contrary that $c=2,3^{a}+7^{b}=5^{d}+11$. Using $\bmod 3, d$ is odd. Thus, using $\bmod 8, a$ is even, $b$ is odd. Hence applying $\bmod 7, a \equiv 2, d \equiv 1(\bmod 6)$. Consideration of our equation $\bmod 5$ yields $a \equiv 2, b \equiv 1(\bmod 4)$ so that, from $\bmod 16$, $d \equiv 1(\bmod 4)$. Now, using $\bmod 27$ we have $(b, d) \equiv(1,13),(4,7)$ or $(7,1)(\bmod (9,18))$. In particular $b \equiv d \equiv 1(\bmod 3)$. Using $\bmod 19$ we conclude that $(a, b, d) \equiv(2,25,1)(\bmod 36)$. Thus we have a contradiction $\bmod 37$. 
6.0796 Lemma. $(a, b, c, d) \equiv(t, 1, t, 1)$ or $(3,3,5,3)(\bmod 6), \quad t$ arbitrary.

Proof. Using mod 3, $d$ is odd. Further, using mod 8 we have the following table of possibilities $\bmod 2$ :

\begin{tabular}{|cccc|}
\hline$a$ & $b$ & $c$ & $d$ \\
\hline 0 & 0 & 1 & 1 \\
0 & 1 & 0 & 1 \\
1 & 1 & 1 & 1 \\
\hline 6.08 & Table. & $(a, b, c, d) \bmod 2$.
\end{tabular}

Using $\bmod 7$ and $\bmod 9$ successively and referring to Table 6.09 we have the following table of solutions $\bmod 6$, where $0 \leqq t \leqq 5$ :

\begin{tabular}{|llll|}
\hline$a$ & $b$ & $c$ & $d$ \\
\hline$t$ & 1 & $t$ & 1 \\
0 & 2 & 1 & 5 \\
1 & 5 & 5 & 5 \\
2 & 3 & 0 & 3 \\
2 & 5 & 4 & 5 \\
3 & 3 & 5 & 3 \\
4 & 0 & 1 & 3 \\
4 & 2 & 3 & 5 \\
\hline
\end{tabular}

6.09 Table. $(a, b, c, d) \bmod 6$.

Using $\bmod 13$, the lemma follows.

6.095 LEMMA. $a>3$.

Proof. Suppose on the contrary that $a=3,25+7^{b}=3^{c}+5^{d}$. Since $d$ is odd and $d>1$ we have two cases: $d=3, d \geqq 4$.

6.0951 Case. $d=3$. $7^{b}=3^{c}+100$. By Lemma $10, c \geqq 6$ so that $7^{b} \equiv 100(\bmod 729), b \equiv 84(\bmod 243)$. Thus, using $\bmod 1459$ we have $c \equiv 248(\bmod 1458)$, contradicting the fact that $c \equiv 5(\bmod 6)$. Hence $d>3$.

6.0952 Case. $d \geqq 4$. From Lemma 6.0796, $(b, c, d) \equiv(1,3,1)$ or $(3,5,3)(\bmod 6)$. Using $\bmod 13$ we conclude $(b, c, d) \equiv(1,3,1)$ or $(3,5,3)(\bmod (12,6,12))$. Further, using $\bmod 25,(b, c) \equiv(1,15)$ or $(3,5)(\bmod (4,20))$ so that $(b, c, d) \equiv(1,15,1)$ or $(3,5,3)(\bmod (12,60,12))$. Thus, using $\bmod 31$ we have $(b, c, d) \equiv(3,5,3)(\bmod (60,60,12))$. Therefore, using $\bmod 11,(b, c, d) \equiv(3,5,3)(\bmod 60)$. Hence $25+$ 
$7^{b} \equiv 3^{c}, 3^{c} \equiv 118(\bmod 125)$ so that $c \equiv 5(\bmod 100)$. Hence, using $\bmod 101$ and combining results, $(b, c, d) \equiv(3,5,3)(\bmod 300)$. Further $25+7^{b} \equiv 3^{c}, 368 \equiv 3^{c}(\bmod 625)$ so that $c \equiv 205(\bmod 500)$. Hence, using $\bmod 251$ we have a contradiction. Thus $a \geqq 4$.

\subsection{Lemma. $(a, b, c, d) \not \equiv(3,3,5,3)(\bmod 6)$.}

Proof. Suppose the contrary. Using $\bmod 13$ we conclude $b \equiv 3$ $(\bmod 12), d \equiv 3(\bmod 4)$. Hence, using $\bmod 5, c \equiv 1, a \equiv 3(\bmod 4)$. Thus $(a, b, c, d) \equiv(3,3,5,3)(\bmod 12)$. Using $\bmod 27$ we conclude that $(b, d) \equiv(0,9),(3,3)$ or $(6,15)(\bmod (9,18))$. Hence, $(b, d) \equiv(0,27)$, $(3,3)$ or $(6,15)(\bmod (9,36))$. Consideration of our equation $\bmod 37$ yields $(a, b, c, d) \equiv(3,3,5,3)(\bmod 36)$. Therefore, using $\bmod 81$, $(b, d) \equiv(3,39),(12,21)$ or $(21,3)(\bmod (27,54))$. Applying these results $\bmod 109$ we find $(a, b, c, d) \equiv(12,12,5,21)(\bmod 27)$ so that in fact $(a, b, c, d) \equiv(39,39,5,21)(\bmod 54)$. Consideration of cases $\bmod 163$ yields a contradiction. Hence $(a, b, c, d) \not \equiv(3,3,5,3)(\bmod 6)$.

6.0954 Lemma. For any positive integer $t,(a, b, c, d) \not \equiv(t, 1, t, 1)$ (mod 6). (Thus the proof of the theorem is completed.) Note that the equality $(a, b, c, d)=(t, 1, t, 1)$ is excluded in this assertion.

Proof. Suppose the contrary. Using $\bmod 13, b \equiv 1(\bmod 12)$, $d \equiv 1(\bmod 4)$. Hence, using $\bmod 5, a \equiv c(\bmod 4)$. Thus $(a, b, c, d) \equiv$ $(t, 1, t, 1)(\bmod 12)$. Using $\bmod 25$ we have the following table of pairs $(a, c)(\bmod 20)$ :

\begin{tabular}{|r|rrrrrrrrrrrrrrrrrrrrr}
\hline$a$ & 0 & 1 & 2 & 3 & 4 & 5 & 6 & 7 & 8 & 9 & 10 & 11 & 12 & 13 & 14 & 15 & 16 & 17 & 18 & 19 \\
$c$ & 4 & 9 & 18 & 15 & 8 & 13 & 2 & 19 & 12 & 17 & 6 & 3 & 16 & 1 & 10 & 7 & 0 & 5 & 14 & 11 \\
\hline
\end{tabular}

6.10 Table. $(a, c) \bmod 20$.

Applying the above results and using mod 31 we arrive at the following table of possibilities mod 60 :

\begin{tabular}{|ccc|}
\hline$a$ & $b$ & $c$ \\
\hline 25 & 25 & 13 \\
31 & 37 & 43 \\
57 & 49 & 45 \\
58 & 13 & 34 \\
51 & 13 & 3 \\
56 & 25 & 20 \\
38 & 37 & 14 \\
28 & 49 & 22 \\
\hline
\end{tabular}

6.11 Table. $(a, b, c) \bmod 60$. 
Thus, using mod 11 we have the following table of possibilities $(\bmod 60)$ :

\begin{tabular}{|cccc|}
\hline$a$ & $b$ & $c$ & $d$ \\
\hline 25 & 25 & 13 & 13 \\
57 & 49 & 45 & 37 \\
58 & 13 & 34 & 25 \\
51 & 13 & 3 & 49 \\
38 & 37 & 14 & 1 \\
\hline
\end{tabular}

6.12 Table. $(a, b, c, d) \bmod 60$.

In each of the above cases we have a contradiction if we consider our equation $\bmod 61$.

(We note that (6.06) also has solutions in negative integers. It is easily seen (using mod 25) that these are the quadruples $(a, b, c, d)=$ $(t, 1, t, 1), t$ negative.)

7. Equations of the Form $p^{a}+q^{b}+r^{c}=s^{d}$. An equation of the form $p^{a}+q^{b}+r^{c}=s^{d}$ does not admit the trivial solution; but nonetheless it can be difficult to find all of its solutions. However, in many cases the equation is quite tractable.

7.01 THEOREM. The only solutions to

$$
5^{a}+5^{b}+3^{c}=3^{d}
$$

in nonnegative integers are $(a, b, c, d)=(0,1,1,2),(0,2,0,3)$ and $(0,0,0,1)$.

Proof. Let $(a, b, c, d)$ be another solution. Either $a=0$ or $a>0$.

Suppose that $a=0$. Then $1+5^{b}=3^{d}-3^{c}$ and clearly $b>2$. Further $d>c$. There are two cases: $c=0$ and $c>0$.

7.021 Case. $\quad c=0$. Then $2+5^{b}=3^{d} . \quad$ Clearly $d \geqq 4,2+5^{b} \equiv 0$ $(\bmod 81) . \quad b \equiv 20(\bmod 54) . \quad$ This yields a contradiction $\bmod 109$.

7.022 Case. $c>0$. Using $\bmod 3$ we conclude that $b$ is odd. Hence, using mod $8, d$ must be even and $c$ must be odd. Using $\bmod 5, d \equiv 2, c \equiv 1(\bmod 4)$. Thus using $\bmod 25$ we have the following table:

\begin{tabular}{|l|lllll|}
\hline$c$ & 9 & 1 & 13 & 5 & 17 \\
\hline$d$ & 2 & 6 & 10 & 14 & 18 \\
\hline
\end{tabular}

7.03 Table. $(c, d) \bmod 20$. 
Thus, using $\bmod 11,(b, c, d) \equiv(3,9,2)(\bmod (10,20,20)) . \quad$ This gives a contradiction $\bmod 61$.

If, on the other hand $a>0$ (and by symmetry $b>0$ ), it is seen (using $\bmod 5)$ that $c \equiv d(\bmod 4)$. This yields a contradiction $\bmod 8$.

\subsection{THEOREM. The only solutions to}

$$
3^{a}+5^{b}+7^{c}=11^{d}
$$

in nonnegative integers are $(a, b, c, d)=(1,0,1,1)$ and $(2,0,0,1)$.

Proof. Let $(a, b, c, d)$ be another solution. Using the moduli 3 and 8 successively we conclude that $a \neq 0$. Further we have the following table:

\begin{tabular}{|cccc|}
\hline$a$ & $b$ & $c$ & $d$ \\
\hline 0 & 0 & 0 & 1 \\
1 & 0 & 1 & 1 \\
\hline
\end{tabular}

7.06 Table. $(a, b, c, d) \bmod 2$.

The remainder of the proof requires the following three lemmas.

LEMMA 7.041. $b=0$.

Proof. Assume the contrary. Using mod 5 we conclude that $a \equiv 1, c \equiv 3(\bmod 4)$. Thus, using $\bmod 7$ we have the following table:

\begin{tabular}{|rll|}
\hline$a$ & $b$ & $d$ \\
\hline 1 & 0 & 1 \\
3 & 4 & 3 \\
5 & 2 & 5 \\
\hline 7.07 & Table. & $(a, b, d) \bmod 6$. \\
\hline
\end{tabular}

Using the moduli 9, 13 successively in each case we have a contradiction. Hence $b=0$.

LEMMA 7.042. $c>0$.

Proof. Suppose that $c=0$. Then $3^{a}+2=11^{d}$. Clearly $a \geqq 4$ so that $2 \equiv 11^{d}(\bmod 81), d \equiv 25(\bmod 54)$. Using $\bmod 19, a \equiv 2$ (mod 18). Hence using $\bmod 109$ we have a contradiction. Thus $c>0$. 
LEMMA 7:043. $a>1$.

Proof. Suppose $a=1$. Then $4+7^{c}=11^{d}$. Clearly $c>1$ so that $4 \equiv 11^{d}(\bmod 49), \quad d \equiv 16(\bmod 21), \quad$ and, in fact $d \equiv 37(\bmod 42)$. Hence, using $\bmod 9, c \equiv 1(\bmod 3)$ so that $($ from Table 7.06$) c \equiv 1$ $(\bmod 6)$. We immediately have a contradiction $\bmod 43$. Hence $a>1$.

To complete the proof of the theorem, observe that, using mod 7, $(a, d) \equiv(0,5)$ or $(1,1)(\bmod 6)$. Thus, using $\bmod 9,(a, c, d) \equiv(0,2,5)$ or $(1,3,1)(\bmod 6)$. In each case we have a contradiction $\bmod 13$. Thus $(a, b, c, d)$ does not exist.

7.08 THEOREM. The only integral solution of

$$
5^{a}+5^{b}+7^{c}=17^{d}
$$

is $(a, b, c, d)=(1,1,1,1)$.

Proof. Let $(a, b, c, d)$ be another solution. Clearly $a, b, c, d \geqq 0$. Using $\bmod 8$ we conclude that $(a, b, c) \equiv(0,0,1)$ or $(1,1,1)(\bmod 2)$. Hence using $\bmod 3, a \equiv b \equiv c \equiv d \equiv 1(\bmod 2)$. Thus, using the moduli 7,9 successively we have $(a, b, c, d) \equiv(1,1,1,1),(3,3,3,5)$ or $(5,5,5,3)(\bmod 6)$. Using $\bmod 16$ we find that $a \equiv b(\bmod 4)$. Also, using $\bmod 5, c \equiv d(\bmod 4)$. Thus, using $\bmod 13$ we have $(a, b, c, d) \equiv(1,1,1,1)$ or $(5,5,11,3)(\bmod 12)$. Then, using $\bmod 31$ we find $(a, b, d) \equiv(1,1,1),(1,1,19)$ or $(5,5,27)(\bmod (12,12,30))$ so that in fact $(a, b, c, d) \equiv(1,1,1,1),(1,1,1,9)$ or $(5,5,11,7)$ $(\bmod (12,12,12,20))$. Hence using $\bmod 25, a=b=1$. (Therefore, $c \equiv 1(\bmod 12)$.) $\quad$ Thus $10+7^{c}=17^{d}, c \geqq 2$, so that $10 \equiv 17^{d}(\bmod 49)$, $d \equiv 19(\bmod 42)$. Finally we have a contradiction $\bmod 43$.

\subsection{THEOREM. The equation}

$$
2^{a}+2^{b}+7^{c}=29^{d}
$$

has no integral solution.

Proof. Let $(a, b, c, d)$ be a solution. Clearly $a, b, c, d \geqq 0$. By consideration of cases using $\bmod 7$ and $\bmod 3$ we easily conclude that $a, b \geqq 4$. Hence, using $\bmod 16, c \equiv 0(\bmod 2), d \equiv 0(\bmod 4)$. Thus using $\bmod 3, a \not \equiv b(\bmod 2)$. Therefore, using $\bmod 5$ we conclude (without restricting generality) that $a \equiv 3, b \equiv 2, c \equiv 2(\bmod 4)$. In particular, $c>0$ so that consideration of our equation $\bmod 7$ yields $a \equiv b \equiv 2(\bmod 3)$. Hence $a \equiv 11, b \equiv 2(\bmod 12)$. Thus $7^{c} \equiv 2^{d}$ 
$(\bmod 9)$ so that $(c, d) \equiv(0,0),(1,4)$ or $(2,2)(\bmod (3,6))$. Hence $(c, d) \equiv(6,0),(10,4)$, or $(2,2)(\bmod (12,6))$ so that we have a contradiction $\bmod 13$.

7.12 THeOREM. The equation

$$
2^{a}+3^{b}+5^{c}=n^{d}
$$

where $n \equiv 7(\bmod 150)$ and $a, b, c, d$ are nonnegative integers has (i) only the solution $(a, b, c, d)=(0,0,1,1)$ if $n=7$; (ii) no solutions if $n>7$.

Proof. Suppose $n \equiv 7(\bmod 150)$ and $(a, b, c, d)$ is a nonnegative solution of (7.13). Clearly $a=0$. Further, if $b=0$ and $n>7$ then $2 \equiv 7^{d}(\bmod 25)$, an impossibility. In the remaining case, $b>0$, we have a contradiction mod 3 .

7.14 THEOREM. The equation

$$
3^{a}+5^{b}+7^{c}=n^{d}
$$

has no nonnegative integral solution if $n \equiv 19(\bmod 24)$.

Proof. Let $(a, b, c, d)$ be a solution. Using mod 3 we conclude that $a=0, b$ odd. Thus, using $\bmod 8$ we have a contradiction.

7.16 COROLLARY. Equation 7.15 has no solution for the primes $n=19,43,67,139$ and 163 .

The following general theorem is obvious.

7.17 THEOREM. The equation

$$
p^{a}+q^{b}+r^{c}=s^{d}
$$

has no solution in nonnegative integers if $p, q, r, s \equiv 1(\bmod m)$ for some integer $m>2$.

8. Problems and conjectures. In this section we list some conjectures that are suggested by the results of this article.

8.01 Problem. Let $k$ be a given integer, $\varepsilon_{i}= \pm 1$. Suppose that distinct primes $p_{i}$ are also given. Show that all nonnegative integral solutions of the equation

$$
\sum \varepsilon_{i} p_{i}^{a(i)}=k
$$


can be found by reducing (8.02) to a finite number of congruences with respect to certain integral moduli $m_{j}, j=1,2, \cdots, r$.

8.03 PROBLem. If $p_{1}=p_{2}$ but the hypotheses of 8.01 are otherwise unchanged, what is the situation?

8.031 Comment. It is not difficult to show that for any finite set of moduli $\left\{m_{i}\right\}$ the equation

$$
2^{a}+3^{b}=2^{c}+3^{d}
$$

which has the obvious solutions $a=c, b=d$ and the exotic solutions $(a, b, c, d)=(2,0,1,1),(4,0,3,2),(5,1,3,3)$ or $(8,1,4,5)($ with $a>c)$ cannot be fully investigated by reducing it to the congruences $2^{a}+3^{b} \equiv 2^{c}+3^{d}\left(\bmod m_{i}\right)$. However it seems likely that (8.032) has no more solutions.

8.033 Comment. Similar remarks apply to the equation

$$
p^{a}+q^{b}=p^{c}+q^{d}
$$

where $p$ and $q$ are any given distinct primes.

8.035 Comment. The equation

$$
3^{a}=1+2^{b}+2^{c}
$$

was brought to our attention by R. L. Graham. The equation is a special case of one solved by Pillai [10]. Moreover, using a few small moduli, L. J. Alex [2b] established that the only solutions are $(a, b, c)=(1,0,0),(2,2,2),(4,6,4)$ or $(4,4,6)$.

8.037 Comment. The class of equations

$$
1+(p q)^{a}=p^{b}+q^{c}
$$

where $p, q$ are given distinct primes, does not seem to be amenable to our methods.

8.039 Comment. As is demonstrated in this article, there are many equations of the form (8.02) with $p_{1}=p_{2}$ which are solvable by our methods.

8.04 PROBLEM. If the bases $p_{i}$ in 8.01 are replaced by arbitrary pre-assigned pairwise relatively prime integers, can (8.02) be solved using a finite set of moduli? 


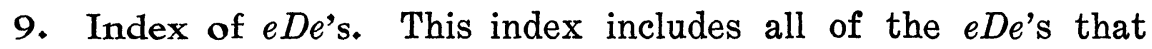
have been brought to our attention. There are many others that can be solved by transparent arguments. (See §1.)

\section{Two term equations}

$x^{y}=y^{x}$

$x^{y^{z}}=z^{x^{y}}$

[4], [7]

(We know no reference.)

Three term equations

$y=1+x$ ( $x y$ divisible by no primes except $2,3,5$ )

$z=x+y$ ( $x y z$ divisible by no primes except $2,3,5,7$ )

[1], [8], [9]

Four term equations-three terms on one side

$$
\begin{aligned}
& 3^{a}=1+2^{b}+2^{c} \\
& 3^{d}=3^{c}+5^{a}+5^{b} \\
& 11^{d}=3^{a}+5^{b}+7^{c} \\
& 17^{d}=5^{a}+5^{b}+7^{c} \\
& 29^{d}=2^{a}+2^{b}+7^{c} \\
& n^{d}=2^{a}+3^{b}+5^{c}(n \equiv 7(\bmod 150)) \\
& n^{d}=3^{a}+5^{b}+7^{c}(n \equiv 19(\bmod 24), \text { e.g., } n=19,43,67,139,163) \\
& s^{d}=p^{a}+q^{b}+r^{c}(p \equiv q \equiv r \equiv s \equiv 1(\bmod m), m>2)
\end{aligned}
$$

Four term equations-two terms on each side

$$
\begin{aligned}
& 1+2^{a}=2^{b}+3^{c} \\
& 1+2^{a}=2^{b}+5^{c} \\
& 1+2^{a}=2^{b}+7^{c} \\
& 1+2^{a}=4 \cdot 3^{b}+5^{c} \\
& 1+3^{a}=5^{b}+7^{c} \\
& 1+5^{a}=3^{b}+7^{c} \\
& 1+5^{a}=3^{b}+3^{c} \\
& 1+5^{a}=3^{b}+23^{c} \\
& 1+5^{a}=7^{b}+7^{c} \\
& 1+5^{a}=7^{b}+19^{c} \\
& 1+5^{a}=11^{b}+11^{c} \\
& 1+5^{a}=17^{b}+17^{c} \\
& 1+5^{a}=2 \cdot 3^{b}+3 \cdot 2^{c} \\
& 1+7^{a}=3^{b}+5^{c} \\
& 1+13^{a}=17^{b}+17^{c} \\
& 1+17^{a}=7^{b}+7^{c} \\
& 1+67^{a}=17^{b}+17^{c} \\
& 1+p^{a}=q^{b}+r^{c}(q \equiv 1(\bmod p)) \\
& 1+(p q)^{a}=p^{b}+q^{c} \\
& 9+5^{a}=3^{b}+7^{c} \\
& 2^{a}+3^{b}=2^{c}+3^{d}
\end{aligned}
$$




$$
\begin{aligned}
& 3^{c}+5^{d}=5^{a}+7^{b} \\
& 3^{a}+5^{b}=11^{c}+7^{d} \\
& 3^{c}+5^{d}=13^{a}+7^{b} \\
& 3^{c}+5^{d}=17^{a}+7^{b} \\
& 3^{c}+5^{d}=m^{a}+7^{b}\left(m=3 \cdot 5^{2} \cdot 7 \cdot 11 \cdot r, r \equiv-1(\bmod 8)\right) \\
& 3^{c}+5^{d}=m^{a}+7^{b}\left(m \equiv 1\left(\bmod 2^{4} \cdot 3^{2} \cdot 5^{2} \cdot 7 \cdot 13\right)\right) \\
& 3^{c}+7^{d}=5^{a}+7^{b} \\
& 3^{c}+7^{d}=5^{a}+7^{b} \\
& 3^{a}+7^{b}=5^{c}+11^{d} \\
& 3^{c}+7^{d}=5^{a}+15^{b} \\
& 3^{c}+7^{d}=n^{a}+5^{b}(n \equiv 1(\bmod 6), n \equiv \pm 1(\bmod 5), n \neq \equiv \pm 1(\bmod 8) \\
& \text { simultaneously; e.g., } n=19,61,109,139,181 \\
& \text { or } n \equiv 19(\bmod 120)) \\
& 3^{c}+7^{d}=n^{a}+5^{b}(n \equiv 1(\bmod 6), n \equiv \pm 2(\bmod 5), n \neq \equiv \pm 1(\bmod 8) \\
& \text { simultaneously; e.g., } n=13,37,43,67,133 \text {, } \\
& 157 \text { or } n \equiv 13(\bmod 120)) \\
& 3^{c}+7^{d}=n^{a}+5^{b}(n \equiv 1 \text { or } 13(\bmod 42), n \neq \equiv \pm 1(\bmod 8) \\
& \text { simultaneously; e.g., } n=211,307,349,379 \text {, } \\
& 421,547,643,757 \text { or } n \equiv 13(\bmod 168)) \\
& 3^{c}+7^{d}=n^{a}+5^{b}(n \equiv 17(\bmod 120)) \\
& 3^{a}+11^{b}=5^{c}+7^{d} \\
& 3^{d}+43^{c}=7^{b}+43^{a} \\
& 3^{b}+n^{a}=5^{c}+7^{d}(n \equiv 1(\bmod 6), n \not \equiv 0(\bmod 5), n \not \equiv \pm 1(\bmod 8) \\
& \text { simultaneously; e.g., } n=13,19,37,43,61 \\
& \text { or } n \equiv 13(\bmod 120)) \\
& p^{a}+q^{b}=p^{c}+r^{d}(p \equiv q \equiv 1(\bmod r), p \equiv 1(\bmod q) \\
& \text { simultaneously) } \\
& p^{a}+p^{b}=p^{c}+p^{d} \\
& p^{a}+p^{b}=q^{c}+q^{d}(q \equiv 1(\bmod p))
\end{aligned}
$$

Equations with more than four terms

$$
\begin{aligned}
& 3^{c}+5^{d}=1+2^{a}+7^{b} \\
& 3^{a}+7^{b}=2+3^{c}+5^{d} \\
& \sum \varepsilon_{i} p_{i}^{a(i)}=k
\end{aligned}
$$

Acknowledgment. We thank Herman Robinson and L. J. Alex for checking many of our computations, thus reducing the frequency of our errors.

\section{REFERENCES}

1. L. J. Alex, Diophantine equations related to finite groups, Comm. in Algebra, 4 (1), (1976), 77-100.

2. The Diophantine equation $3^{a}+5^{b}=7^{c}+11^{d}$, Notices of the Amer. Math. Soc., 26 (5), (1979), A-454, 768-10-13. 
2a. L. J. Alex, Private communication.

2b. , Private communication.

2c. - On Simple Groups of Order $2^{a} 3^{b} 7^{c} p$, Journal of Algebra, 25 (1) (1973), 113-124.

2d. Simple Groups of Order $2^{a} 3^{b} 5^{c} 7^{d} p$, Transactions of the Amer. Math. Soc., 173 (1972), 389-399.

3. , Problem S 31, Amer. Math. Monthly, 87 (2) (1980), 403.

4. L. E. Dickson, History of the theory of numbers, Washington, 2 (1920), 687.

5. L. L. Foster, Solution to problem E2749, Amer. Math. Monthly, 87 (2), (1980), 138.

6. - Solution to problem E2750, Amer. Math. Monthly, 87 (2), (1980), 138-139.

7. M. Gardner, Mathematical games, Scientific American, 241 (3), (1979), 25.

8. D. H. Lehmer, On a problem of Stormer, Illinois J. Math., 8 (1), (1964), 57-79.

9. S. S. Pillai, On $a^{X}-b^{Y}=b^{y} \pm a^{x}$, J. Indian Math. Soc., (N. S.), 8 (1944), 10-13.

10. On the equation $2^{x}-3^{y}=2^{X}+3^{Y}$, Bull. Calcutta Math. Soc., 37 (1945), $15-20$.

11. H. P. Schlickewei, Über die diophantische Gleichung $x_{1}+x_{2}+\cdots+x_{n}=0$, Acta Arith., 33 (1977), 183-185.

12. C. Størmer, Quelques théorèmes sur l'equation de Pell $x^{2}-D y^{2}=1$ et leurs applications, Skrifter Videnskabs-seskabet (Christiana), I, Mat. Naturv. K1., (1897), no. 2 (48 pp.).

13. M. Voorhoeve, K. Györy, and R. Tijdeman, On the diophantine equation $1^{k}+2^{k}+$ $\cdots+x^{k}+R(x)=y^{z}$, Acta Math., 143 (1979), 1-8.

Received November 6, 1980.

10 Phillips Road

Palo Alto, CA 94303

AND

California State University

Northridge, CA 91330 
